OPEN ACCESS

Edited by:

Changbin Chen

University of Minnesota, USA

Reviewed by:

Tao Sun,

Stanford University, USA Sanjay K. Gupta,

University of Minnesota, USA

${ }^{*}$ Correspondence:

Qing Yang

qyang19@njau.edu.cn

Specialty section

This article was submitted to Plant Genetics and Genomics,

a section of the journal

Frontiers in Plant Science

Received: 04 February 2016

Accepted: 26 March 2016

Published: 19 April 2016

Citation:

Tang R, Zhu W, Song X, Lin X, Cai J,

Wang $M$ and Yang $Q$ (2016)

Genome-Wide Identification and

Function Analyses of Heat Shock

Transcription Factors in Potato.

Front. Plant Sci. 7:490.

doi: 10.3389/fpls.2016.00490

\section{Genome-Wide Identification and Function Analyses of Heat Shock Transcription Factors in Potato}

\author{
Ruimin Tang, Wenjiao Zhu, Xiaoyan Song, Xingzhong Lin, Jinghui Cai, Man Wang and \\ Qing Yang*
}

Biochemistry and Molecular Biology, College of Life Sciences, Nanjing Agricultural University, Nanjing, China

Heat shock transcription factors (Hsfs) play vital roles in the regulation of tolerance to various stresses in living organisms. To dissect the mechanisms of the Hsfs in potato adaptation to abiotic stresses, genome and transcriptome analyses of Hsf gene family were investigated in Solanum tuberosum L. Twenty-seven StHsf members were identified by bioinformatics and phylogenetic analyses and were classified into A, B, and $\mathrm{C}$ groups according to their structural and phylogenetic features. StHsfs in the same class shared similar gene structures and conserved motifs. The chromosomal location analysis showed that $27 \mathrm{Hsfs}$ were located in 10 of 12 chromosomes (except chromosome 1 and chromosome 5) and that 18 of these genes formed 9 paralogous pairs. Expression profiles of StHsfs in 12 different organs and tissues uncovered distinct spatial expression patterns of these genes and their potential roles in the process of growth and development. Promoter and quantitative real-time polymerase chain reaction (qRT-PCR) detections of StHsfs were conducted and demonstrated that these genes were all responsive to various stresses. StHsf004, StHsf007, StHsf009, StHsf014, and StHsf019 were constitutively expressed under non-stress conditions, and some specific Hsfs became the predominant Hsfs in response to different abiotic stresses, indicating their important and diverse regulatory roles in adverse conditions. A co-expression network between StHsfs and StHsf-co-expressed genes was generated based on the publicly-available potato transcriptomic databases and identified key candidate StHsfs for further functional studies.

Keywords: heat shock transcription factors, potato, bioinformatics, abiotic stresses, gene expression, co-expression network

\section{INTRODUCTION}

Plants always suffer from various adverse environmental stresses in their growth and development periods and have developed special mechanisms to respond to the changing adverse conditions (Wang et al., 2014). These response mechanisms are regulated by substantial regulatory interactions and coordination of distinct signal transduction pathways in plant responses to intricate abiotic stresses (Singh et al., 2002; Katagiri, 2004; Ahuja et al., 2010; Mittler et al., 2012).

Numerous signaling components and downstream effectors participate in different biological reactions and connect the reaction pathways into a regulatory network. Among these components, various transcription factors play central roles in regulation of the stress-induced responses 
(Kotak et al., 2007a). Heat shock transcription factors (Hsfs), one sort of the most important transcription regulators, are the terminal elements of the signal transduction chain and mediate the activation of genes responsive to various abiotic stresses including heat stress, drought stress and a large number of chemical stressors (such as $\mathrm{Cd}^{2+}$ and salicylate) (Baniwal et al., 2004). Hsfs can regulate the transcription of Hsp genes by binding specifically with heat shock elements (HSE) in Hsp gene promoters, and Hsps subsequently protect cells against stress impairment and participate in protein folding (Morimoto, 1993; Schöffl et al., 1998; Hartl and Hayer-Hartl, 2002). Several results have already confirmed that Hsfs are responsible to other abiotic stresses apart from heat stress. For instance, HsfAle, $H s f A 3, H s f A 4 a, H s f B 2 a$, and $H s f C 1$ are strongly induced by cold, salt and osmotic stresses in Arabidopsis, suggesting that Hsfs are significant regulatory molecules in the complex network of response pathways (Miller and Mittler, 2006; Kilian et al., 2007; Swindell et al., 2007; Zhang et al., 2015).

Analogous to other transcription factors, a typical feature at the N-terminus of Hsfs with a helix-turn-helix structure is their DNA-binding domain (DBD), which is the best preserved motif and can be used to accurately recognize the core repeating units of HSE (5'-nGAAn-3') (Damberger et al., 1994; Döring et al., 2000; Ma et al., 2014). Another conserved domain adjacent to DBD motif is the oligomerization domain (OD). This domain consists of hydrophobic heptad repeats (HR-A and HR-B) which can form a curly structure to activate the formation of an Hsf trimer to combine with $H s p$ genes' promoter efficiently (Sorger and Nelson, 1989; Wu, 1995; Lyck et al., 1997; Scharf et al., 2012). Besides DBD and OD, a nuclear localization signal (NLS) characterized by a cluster of arginine and lysine residues and a nuclear export signal (NES) rich in leucine residues are two structures close to the C-terminal of Hsfs (Lyck et al., 1997; Scharf et al., 2012). In most class A Hsfs, there is a C-terminal activation domain (CTAD) with amino acids activation sequence (AHA), in which the first " $A$ " represents aromatic amino acids W, F, Y, " $H$ ” represents large hydrophobic amino acids L, I, V, M, and the second " $A$ " stands for acidic amino acids D and $\mathrm{E}$ (Xue et al., 2014). Instead, nearly all class B members in the C-terminal region contain a peptide with four amino acids: -LFGV-, which is considered as the core of a repression sequence and widely exists in other transcription factors, like $A B I 3 / V P 1$ and $M Y B / G R A S$ (Ikeda and Ohme-Takagi, 2009), but how this peptide interacts with other domains and how to play a repression role in the process of transcription remain obscure (Czarnecka-Verner et al., 2004).

Since the development of the sequencing technology, Hsf members from various species have been identified, such as yeast (Wiederrecht et al., 1988), Arabidopsis (Nover et al., 2001), tomato (Mishra et al., 2002), rice (Wang et al., 2009), and pepper (Guo et al., 2015), which contains 1, 21, 24, 25, and 25 members, respectively. So far, only four kinds of Hsfs were discovered in animals, including Hsf1, Hsf2, Hsf3, and Hsf4 (Zheng et al., 2008; Akerfelt et al., 2010). Compared with the animal Hsfs, plant Hsfs are more diverse, partially redundant, and functionally flexible (Miller and Mittler, 2006). The components of Hsf members have noticeable difference among different plant species. Although Hsfs in Dicotyledonous and monocotyledonous have high similarity, some types of Hsfs express specifically: HsfA9 only exists in dicotyledonous plants and HsfC2 only exists in monocotyledonous plants (Xue et al., 2014). Even though the composition of Hsfs in different plants is clear, the functions of these Hsfs have not been determined completely.

Many researches have demonstrated that the function of Hsf members differs from the species in plant development and in the interlaced stress response pathways (Xue et al., 2014). HsfA3 has been found as an important player in the crosstalk of heat and drought stresses in Arabidopsis while similar function of HsfA3 was not detected in tomato (Von Koskull-Döring et al., 2007). HsfA9 was considered to play a specific role in seed development in many plant species like Arabidopsis, tomato and tobacco whereas HsfA7 appears to perform the parallel function as HsfA9 in rice (Kotak et al., 2007b; Von Koskull-Döring et al., 2007; Chauhan et al., 2011). Although HsfA9 was well known in regulating the seed maturation, it has also been described to involve in the development of pericarp and placenta in pepper (Guo et al., 2015).

Multiple signal transduction pathways have been demonstrated to regulate the Hsp expression by activating Hsfs to bind to the heat shock element of heat shock genes, but the molecular pathway is still elusive for the entire function of Hsfs and their related genes in different stresses (Singh et al., 2002; Zhang et al., 2015). Recently, the package of WGCNA (Weighted correlation network analysis) has provided a possible method to analyze the response of Hsfs and the correlated genes to different abiotic stresses using numerous microarray datasets or transcription data (Langfelder and Horvath, 2008). WGCNA package, which was designed for clustering the related genes into a module based on the tissue types and correlated biological pathways, has been used in mouse, yeast and many kinds of plant species (Langfelder and Horvath, 2008). By this way, Downs et al. (2013) identified several tissue-specific modules and signal pathway-specific modules in maize, and also detected the potential molecular components in these modules. Zhang et al. (2015) created a co-expression network using this package to investigate the relationship between Hsfs and Hsps in Populus and discover the possible regulatory mechanisms among them.

Potato is the fourth most important food crop following wheat, corn and rice in the world. During the potato field cultivation, adverse stresses often do great harm to their growth and lead to a decline in potato output. Therefore, study on stress resistance and exploitation of related genes is becoming more and more important and urgent for potato breeding and production. To our knowledge, there are no reports for identification and functional elucidation of potato Hsfs to date. In this study, a bioinformatics analysis was used to conduct the genome-wide identification of potato Hsf family members with the open access databases. In order to shed light into their underlying roles, the expression profiles of these deduced Hsf members were performed in various tissues and also in response to heat and other abiotic stresses (drought and cold stress). Our analysis indicated that some Hsf genes exhibited specific expression patterns in response to distinct stresses. A co-expression network 
between StHsfs and their correlated genes helped to identify molecular components in the same pathways and select the candidate genes for further research.

\section{MATERIALS AND METHODS}

\section{Plant Materials and Growth Conditions}

The potato plantlets (Solanum tuberosum L.) from our own laboratory were cultured in MS medium (Murashige and Skoog, 1962) containing 3\% sucrose and $0.8 \%$ agar at $\mathrm{pH} 5.8$, and maintained under $16 \mathrm{~h}$ light $/ 8 \mathrm{~h}$ dark regime at $22 \pm 1{ }^{\circ} \mathrm{C}$. Subculture was conducted every 4 weeks. The 1 -month-old plantlets were then transferred into tubes with half strength Hoagland solution with aeration in illumination incubator under $16 \mathrm{~h}$ light $/ 8 \mathrm{~h}$ dark regime at $22 \pm 1^{\circ} \mathrm{C}$ for another 2 weeks before being treated with heat, drought and cold stresses. For temperature stress treatments, the plantlets were exposed to 4 or $35^{\circ} \mathrm{C}$; for drought treatments, the plantlets were incubated with 3\% PEG-6000. Under these different stress treatments, the 2nd fully expanded leaves from the top of plantlets leaves were collected at $0,2,6$, and $24 \mathrm{~h}$. All harvested samples were immediately immersed in liquid nitrogen and stored at $-80^{\circ} \mathrm{C}$ prior to RNA extraction.

\section{Identification of the Hsf Members in Potato}

The sequences of potato $H s f$ members were firstly searched in the Plant Transcription Factor Database (Riaño-Pachón et al., 2007). To identify a complete list of potato Hsf genes, the Hidden Markov Model (HMM) profile and consensus pattern of the Hsf DBD (PF00447) were downloaded from the Pfam database (http://pfam.xfam.org/search) to obtain the conserved domain. Protein sequences of Hsfs conserved domain and potato genome protein sequences were aligned by BLASTp research in the NCBI protein database (http://blast.ncbi.nlm. nih.gov/blast.cgi) and Spud DB Potato Genomics Resources (http://solanaceae.plantbiology.msu.edu/) with $E$-value of 0.001 to screen candidate Hsfs with homologous amino acids sequences preliminarily. These candidate genes were analyzed using the domain identification function of the Pfam database $(E=1.0)$ to remove the Hsfs without the conserved domain sequences. The multiple protein sequence alignment of the candidate genes was then performed by Clustal $\mathrm{W}$ which provided by MEGA 4.0 to remove repetitive sequences. Length of amino acids sequences, theoretical molecular weights, isoelectric points and grand average of hydropathicity of deduced Hsf proteins were calculated using ProtParam tools provided by the ExPasy website (http://web.expasy.org/tools/protparam). The DBD and heptad repeat region (HR-A/B) sequences of Hsf proteins were aligned by DNAMAN software, respectively. The information of chromosome location and genomic length of the predicted genes was obtained from the Spud DB Potato Genomics Resources.

\section{Phylogenetic Analysis and Classification of these Identified Hsfs}

The heptad repeat region alignment was used to preliminarily classify these identified Hsfs into three classes. The application of phylogenetic analysis helped to further divide potato Hsf members into different groups based on the Hsf classification scheme of other dicotyledon plants like tomato, cucumber, soybean and Arabidopsis (Scharf et al., 2012; Xue et al., 2014). In total, $145 \mathrm{Hsf}$ protein sequences from potato and other four species (Nover et al., 2001; Baniwal et al., 2004; Chung et al., 2013; Zhou et al., 2013) were used for construction of a phylogenetic tree by Clustal $\mathrm{W}$ alignment and the unrooted Neighbor-Joining method with 1000 bootstrap replicates using MEGA 4.0.

\section{Chromosomal Location of StHsfs}

All identified StHsf genes were mapped to potato chromosomes using MapInspect software (http://www.plantbreeding.wur. $\mathrm{nl} / \mathrm{uk} /$ software-mapinspect.html) based on the information available at the website of Spud DB Potato Genomics Resources (http://solanaceae.plantbiology.msu.edu/). Tandem duplications of paralogous Hsf genes and Hsf gene clusters in the potato genome were marked according to the method used by Yuan et al. (2015) and Zhang et al. (2015), respectively.

\section{Analyses of Gene Structures, Conserved Motifs, and Response Elements}

The exon and intron organization of potato $H s f s$ was depicted by comparing the coding sequences of StHsfs with their corresponding genomic sequences using Gene Structure Display Server online (http://gsds.cbi.pku.edu.cn) (Guo et al., 2007). The program of Multiple Em for Motif Elicitation online (http://meme.sdsc.edu/meme/intro.html) (Bailey et al., 2009) was utilized to analyze the conserved motifs of the predicted StHsf proteins with the following parameters: the number of repetitions, any; the maximum number of motifs, 20; and the optimum width of each motif, between 6 and 300 residues. The analysis of response elements in StHsf genes promoter region (up to $-2000 \mathrm{bp}$ upstream of the coding sequences), which are available in the potato genome database of Phytozome v10.3 (http://www.phytozome.net), was conducted by the PLACE website (http://www.dna.affrc.go.jp/PLACE/) (Higo et al., 1999).

\section{RNAseq Analysis and Co-expression Network Construction}

The expression pattern analysis of StHsf genes in different tissues was carried out using the potato RNAseq data (PGSC, 2011) downloaded from Spud DB Potato Genomics Resources (http://solanaceae.plantbiology.msu.edu/). The normalized expression data from the database were calculated as $\log _{2}$ fold change and displayed in a heatmap using the $\mathrm{R}$ Project software.

A co-expression network between $\mathrm{StH} f \mathrm{~s}$ s and their correlated genes was constructed by weighted correlation network analysis (WGCNA), which provides a systematic method to investigate the potential related genes in the same pathway using the microarray data or RNAseq data (Langfelder and Horvath, 2008). The genes whose maximum expression values in the RNAseq data less than 5 were filtered out. Then the expression data of the remained genes was used to calculate the adjacency matrix between genes in all samples according 
to the Pearson correlation coefficient (Langfelder and Horvath, 2008). The adjacent values between the two genes can be expressed in the following formula (Langfelder and Horvath, 2008):

$$
\mathrm{a}_{i j}=\left|\frac{\left(1+s_{i j}\right)}{2}\right|^{\beta}
$$

In the formula, $a_{i j}$ represents the adjacency value between gene $i$ and gene $j$; $s_{i j}$ is the Pearson correlation coefficient between gene $i$ and $j ; \beta$ represents the weight value. WGCNA method was utilized to convert the adjacency value into the Topological overlap value (TO) which can represent the correlation of the genes in the network. The dissimilarity matrix, the inverse matrix of TO value, was hierarchical clustered to represent the genetic link network. The dynamic treecut algorithm was used to cut the hierarchical clustering tree and the obtained branches represent different modules (Langfelder et al., 2008). The network was graphically presented using Cytoscape software with the TOM (Topological overlap matrix) value 0.07 (Shannon et al., 2003).

\section{RNA Isolation and Expression Analysis of Selected StHsfs Using Quantitative Real-Time PCR}

Total RNA from stress-treated leaves was isolated using the RNA Extract kit (TaKaRa, Dalian, China) with the treatment of RNasefree DNase I to erase the genomic DNA (TaKaRa, Dalian, China). After the examination of the RNA integrity and concentration, $0.5 \mu \mathrm{g}$ RNA per sample was used to synthesize the first-strand cDNA using the Transcriptor First Strand cDNA Synthesis Kit (Roche, Mannheim, Germany). One micro liter of the synthetic cDNA was diluted by $9 \mu \mathrm{L}$ nuclease-free water before the qRTPCR analysis.

Quantitative real-time polymerase chain reaction (qRT-PCR) was carried out with the FastStart Universal SYBR Green Master (ROX) (Roche, Mannheim, Germany) on an ABI 7500 Real Time PCR System (Applied Biosystems, Foster City, CA, USA). The specificity of these primers which designed by Beacon designer software was tested by blast tool in NCBI and dissociation curve analysis. Each PCR reaction was conducted in a $20 \mu \mathrm{l}$ reaction

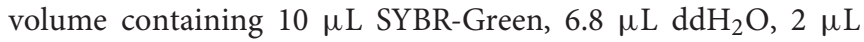
diluted template and $0.6 \mu \mathrm{L} 10 \mu \mathrm{M}$ solution of each primer. The PCR cycling program consisted of $50^{\circ} \mathrm{C}$ for $2 \mathrm{~min}, 95^{\circ} \mathrm{C}$ for $10 \mathrm{~min}$ followed by 40 cycles at $95^{\circ} \mathrm{C}$ for $5 \mathrm{~s}$, and $60^{\circ} \mathrm{C}$ for 35 s. The relative expression of each selected gene was normalized using the method of $2^{-\Delta \Delta \mathrm{Ct}}$ against the reference gene $E F 1 \alpha$ (Yang et al., 2013), whose transcript level keeps relatively steady under different conditions. For each sample, two experimental replicates and three biological repeats were performed to make sure the results reliable. Results were presented as means \pm SD. The method of Dunnett's two-tailed $t$-test was used to conduct the statistical analyses of RT-PCR results, and the statistical significant differences were shown at $p \leq 0.05$ (marked ${ }^{*}$ ) and $p \leq 0.01\left(\right.$ marked $\left.^{* *}\right)$.

\section{RESULTS}

\section{Twenty-Seven Potato Hsf Members Were Identified and Classified into Three Classes}

Potato Hsf members were searched from the Plant Transcription Factor Database, NCBI GenBank database and Spud DB Potato Genomics Resources. Twenty-seven full-length $H s f$ genes were identified as potential members of potato Hsf family after removing the redundant and non-full length sequences according to the consensus pattern of the Hsf DBD in the Pfam database (Supplementary S1). The results of multisequencing alignments of the DBD and heptad repeat regions (HR-A/B) of potato Hsfs presented high conservation in the $\mathrm{DBD}$ and the HR-A/B regions of these Hsf members (Supplementary Figures S1, S2).

Based on the numbers of amino acids inserted in HR-A core and HR-B core of StHsfs, StHsfs were classified into A, $B$, and C three major classes (Supplementary Figure S2). Class A is comprised of 18 proteins, each containing 21 amino acids between HR-A and HR-B except StHsf005 and StHsf006 which respectively have 7 and 5 amino acids deficiency in the insertion region compared with normal HsfA members. Eight StHsfs without amino acids insertion belong to class B, while one StHsf with 7 amino acids between HR-A and HR-B core belongs to class C. These Hsfs were subsequently classified into several subclasses according to the phylogenetic analysis.

All of the 27 putative potato Hsfs were renamed as StHsfoOl to StHsf027 based on the order of class and subclass classification to distinguish them from some existing names such as StHsf8, StHsf24, and StHsf30. The representative information of the StHsf genes and their encoding proteins, containing the genomic length, CDS length, amino acids numbers, the theoretical molecular weight, the isoelectric point and the grand average of hydropathicity, was presented in Table 1. The variation range of protein length of the putative Hsfs were from 201 amino acids (StHsf026) to 501 residues (StHsf005), implying their structure difference and function diversity. The theoretical $\mathrm{pI}$ varying from 4.71 (StHsf014) to 9.58 (StHsf026) indicated that these Hsf proteins may exist and function in different regions of cells. The predicted results of grand average of hydropathicity of these deduced proteins revealed that they all belong to hydrophilic proteins.

\section{Phylogenetic Analysis of Hsfs in Potato and Other Plant Species}

The phylogenetic analysis of potato helped to classify these Hsf members into several subclasses in comparison with the classification scheme of other species which have a close phylogenetic relationship with potato (Figure 1). One small branch marked with an asterisk was unique in potato and classified them into StHsfA8* members because they have high similarity with other members of A8 subclass (Figure 1 and Table 1). In potato, class A and B members were further divided into A1-A9 and B1-B5 subgroups, respectively. Among these subgroups, HsfA1, HsfA4, HsfA6, HsfA7, HsfA8, HsfB2, HsfB3, and HsfB4 were each composed of more than two members; while class $\mathrm{C}$ and other subclasses each contained only one member (Figure 1 and Table 1). 


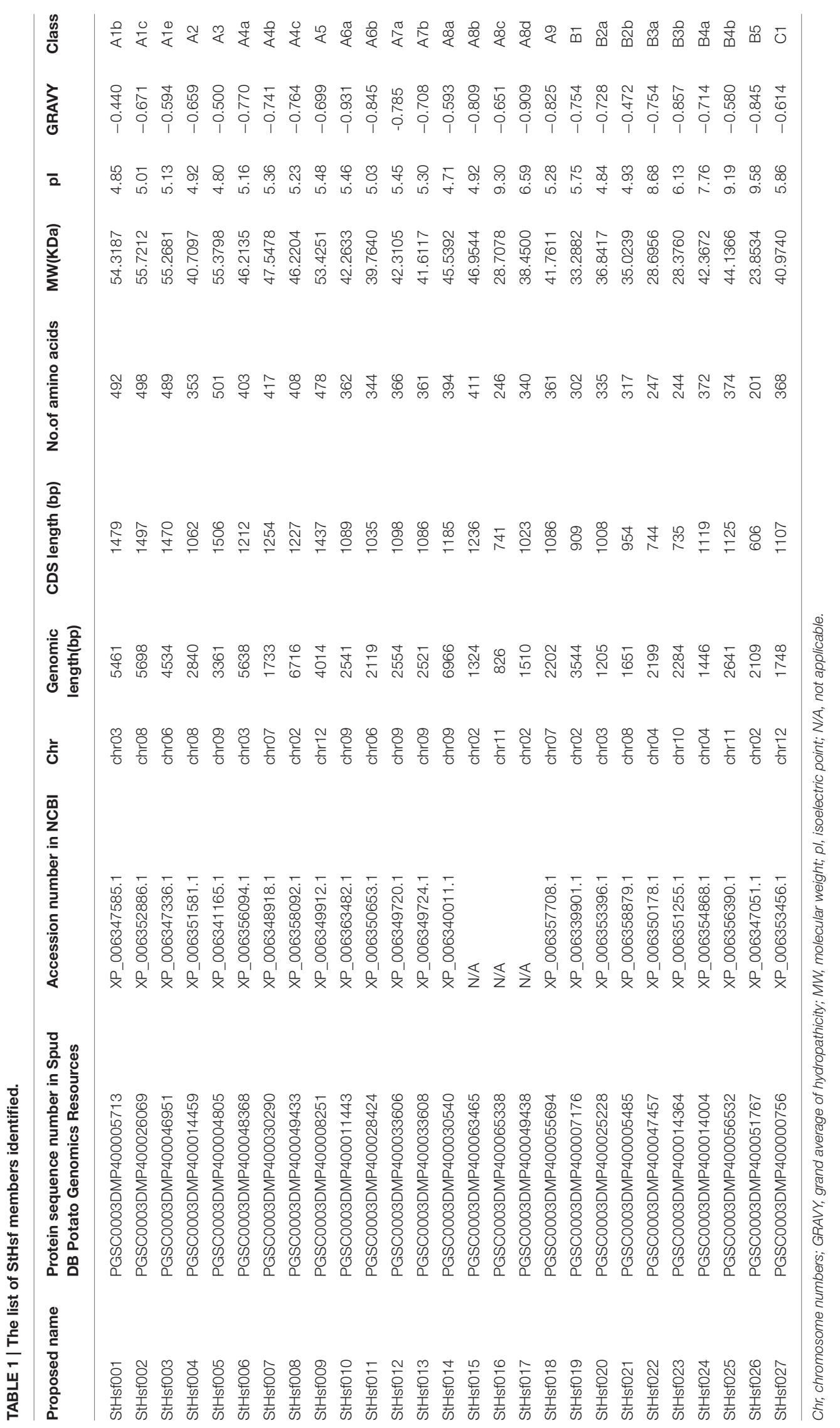




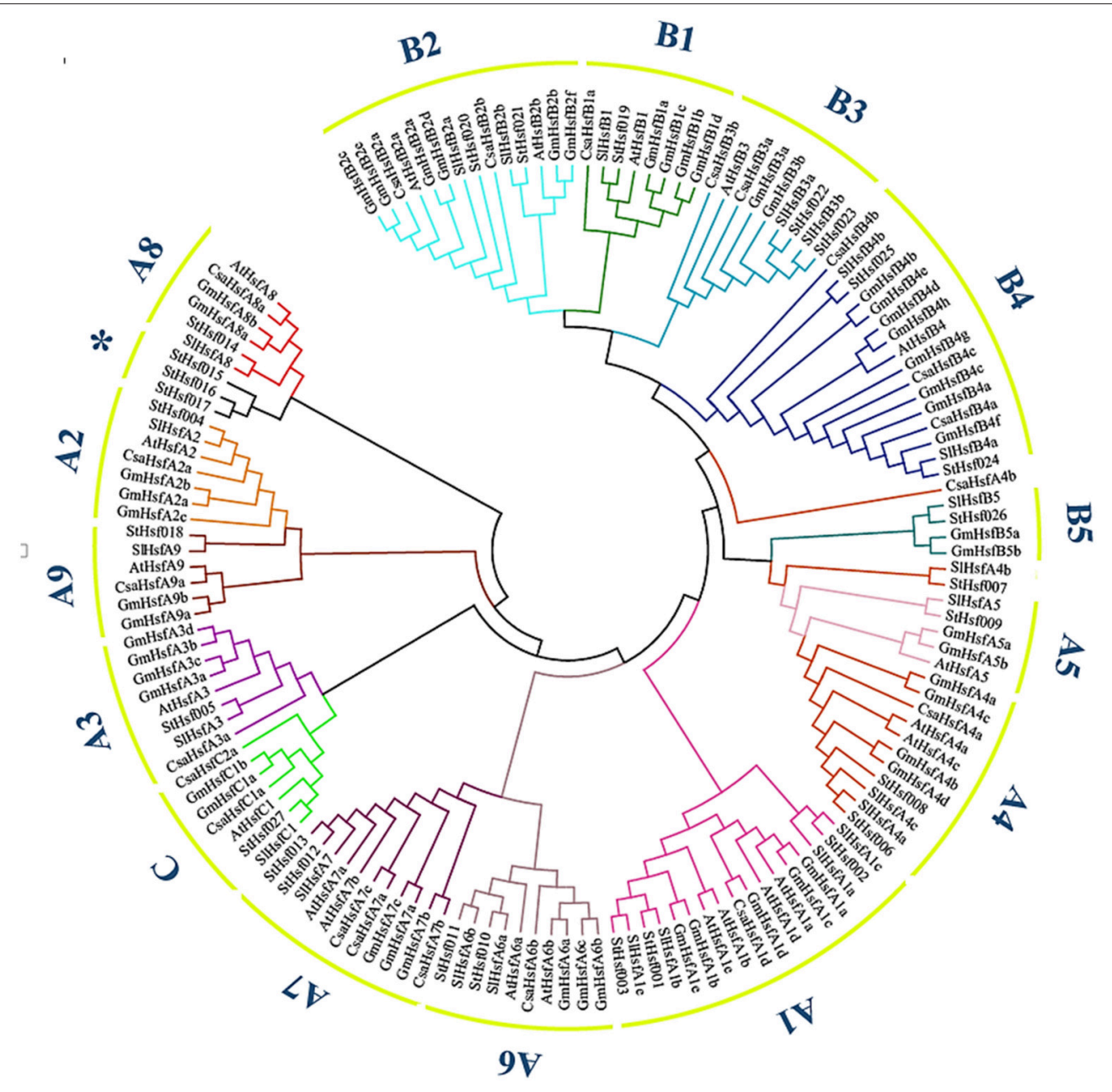

FIGURE 1 | The Neighbor-Joining phylogenetic tree of Hsf proteins from potato, Arabidopsis, tomato, cucumber and soybean. The phylogenetic tree was constructed by Clustal X 2.1 and MEGA 4.0 software using the neighbour-joining option with 1000 bootstrap replicates. Branch lines in different colors represented different subgroups.

The number of StHsfs belonging to each subclass was compared with that in other plant species where this family has been fully identified, such as Arabidopsis (Arabidopsis thaliana) (Nover et al., 2001), tomato (Solanum lycopersicum) (Baniwal et al., 2004), soybean (Glycine max) (Chung et al., 2013), rice (Oryza sativa) (Wang et al., 2009), maize (Zea mays) (Lin et al., 2011), poplar (Populus trichocarpa) (Wang et al., 2012), and pepper (Capsicum annuum) (Guo et al., 2015; Table 2). The composition of Hsfs in potato is similar to Arabidopsis, tomato, soybean, poplar and pepper (all of them are dicotyledonous plants), especially tomato and pepper which are another two kinds of solanaceae plants, but differs significantly from rice and maize, which are monocotyledon. $H s f A 9, H s f B 3$, and $H s f B 5$ are specific genes in dicotyledonous plants indicating that these types might appear after the differentiation of dicotyledonous and monocotyledonous plants. Another detectable difference between monocotyledonary and dicotyledonary Hsfs is that gene duplication in monocot develops a monocotyledon-specific group containing $\mathrm{C} 1 a, C 1 b, C 2 a$, and $C 2 b$ while in most dicotyledon only $C 1$ members exist, which is consistent with the result of Scharf et al. (2012).

\section{Chromosomal Distribution of StHsf Genes}

In order to investigate the distribution of $H s f$ genes on different chromosomes in potato, the location of 27 deduced StHsfs were identified according to the information of potato genome database on Spud DB Potato Genomics Resources. These StHsfs could be mapped to 10 of the 12 potato chromosomes, with no StHsf found on chromosome 1 and chromosome 5 (Figure 2). They unevenly distributed across the chromosomes of the potato genome: Chr10 contains only one $S t H s f$, while relatively high densities of StHsf genes were discovered on Chr02 and Chr09 (5 StHsfs respectively). Most chromosomes contain two (Chr04, Chr06, Chr07, Chr11, and Chr12) or three StHsfs (Chr03 and Chr08).

All of the 27 StHsfs were single copy genes. According to the phylogenetic tree of deduced StHsf sequences (Figure 3A), we linked the paralogous pairs of StHsfs, and found a 
TABLE 2 | Size of the Hsfs classes and subclasses in different plant species.

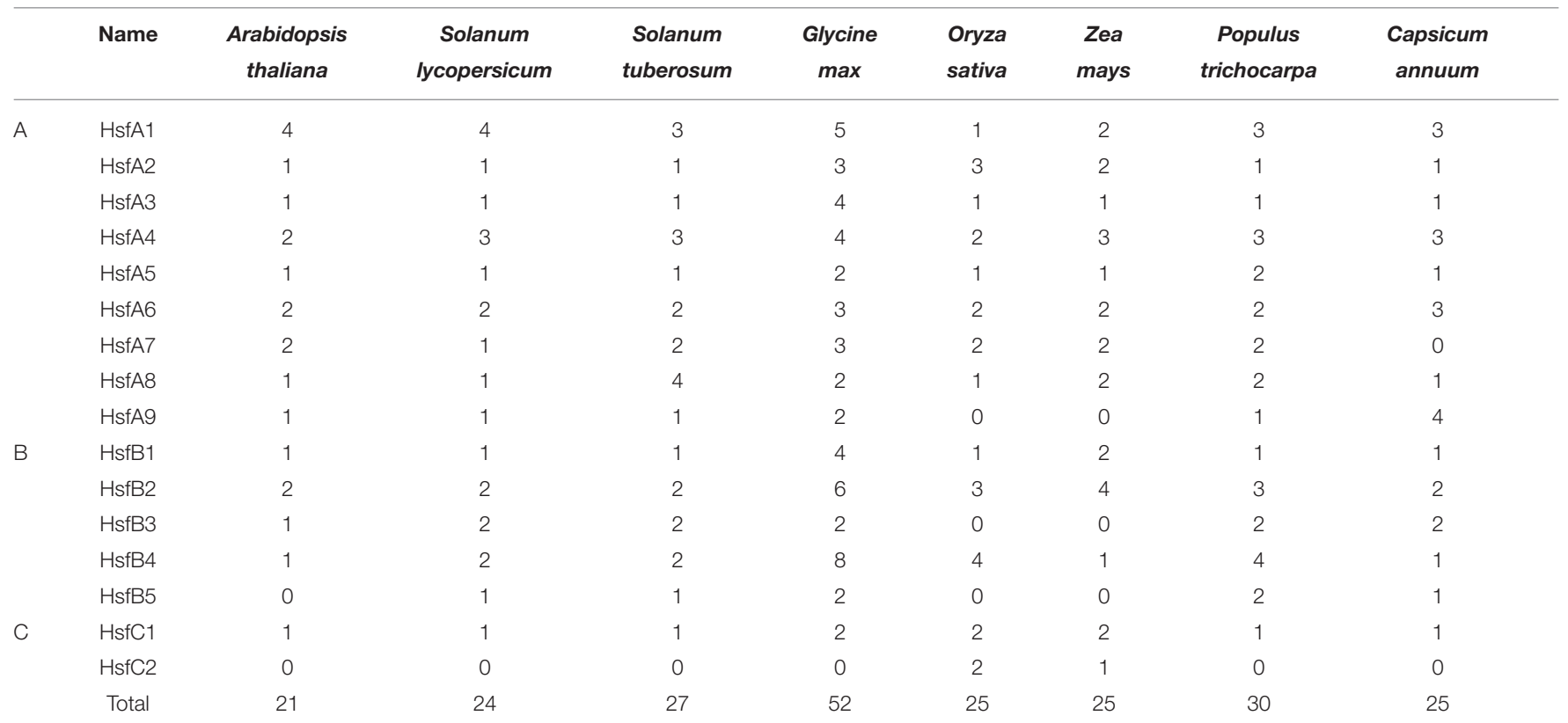

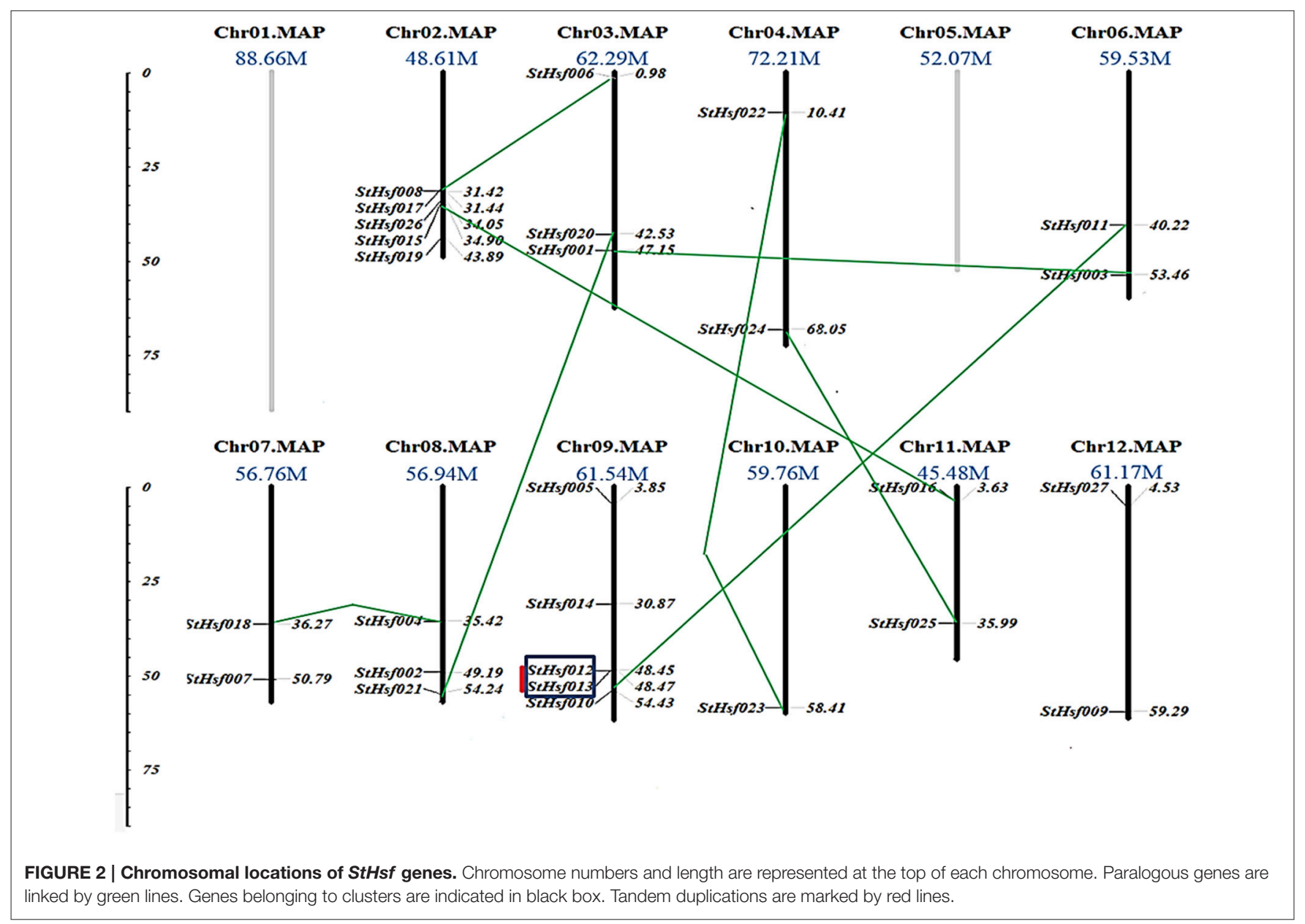




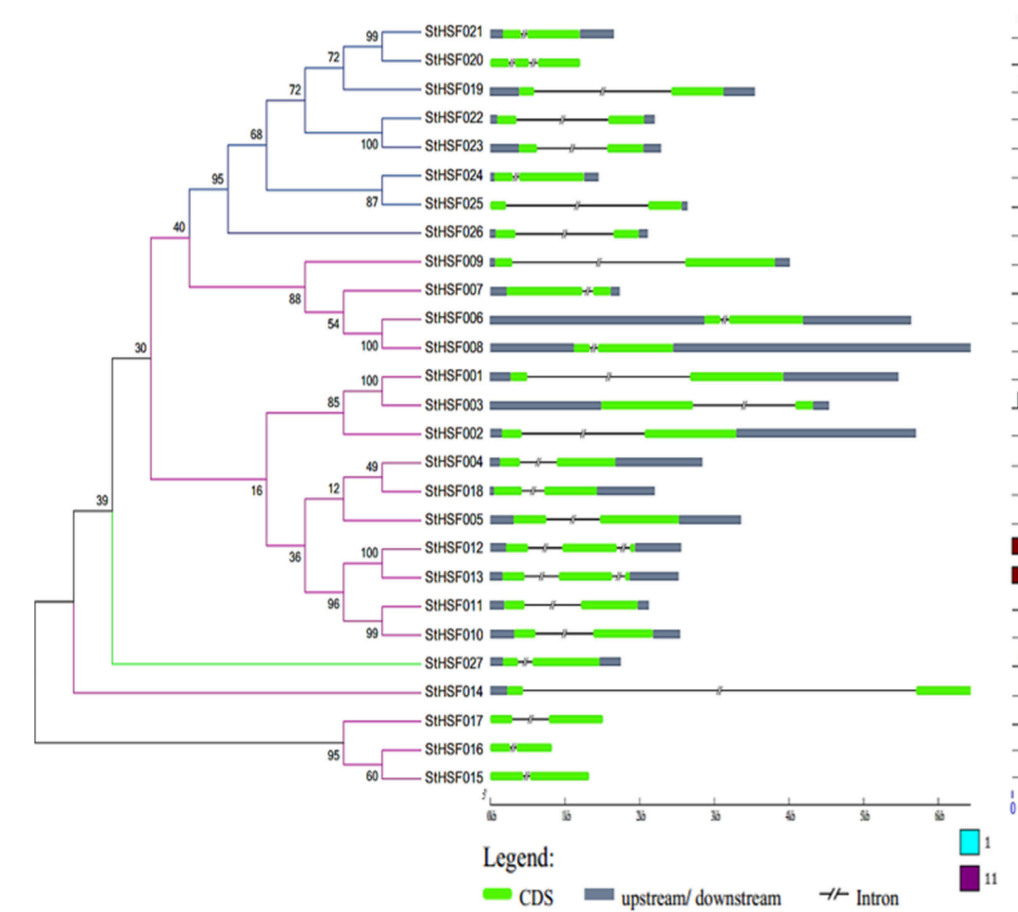

A
B

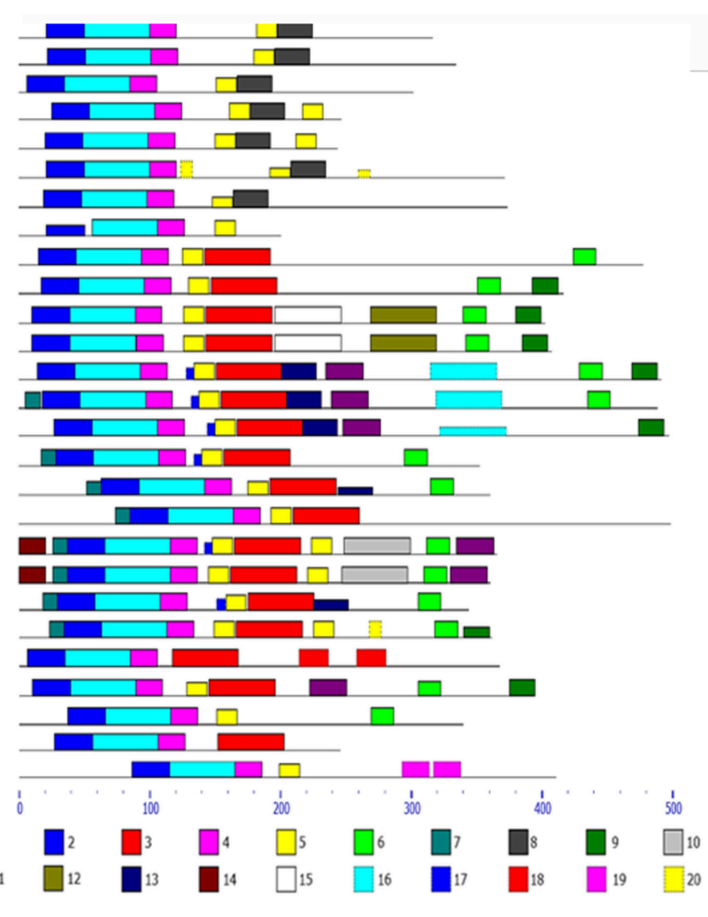

C

FIGURE 3 | Evolutionary relationships (A), gene structures (B) and functional motifs (C) of StHsfs. (A) The phylogenetic tree was constructed by Clustal X 2.1 and MEGA 4.0 software using the neighbour-joining method with 1000 bootstrap replicates. Subtrees branch lines in different colors represent different Hsf classes. (B) The exon/intron distribution of corresponding StHsf genes was detected by comparing these predicted coding sequences (CDS) with their corresponding genomic sequences using GSDS online (http://gsds.cbi.pku.edu.cn). The green boxes represent CDS; the blue boxes indicate upstream or downstream; the discontinuous lines refer to introns of these genes. (C) The motif composition related to each StHsf protein is displayed on the right-hand side. The motifs, numbered 1-20, are displayed in different colored boxes. The sequence information for each motif is presented in Table 3.

total of 9 pairs of paralogous StHsfs: StHsf001/StHsf003, StHsf006/StHsf008, StHsf004/StHsf018, StHsf010/StHsf011, StHsf012/StHsf013, StHsf015/StHsf016, StHsf020/StHsf021, StHsf022/StHsf023, and StHsf024/StHsf025 (Figure 2). Tandem duplications of paralogous genes, defined as two paralogs separated by less than five average-gene-length in the same chromosome, have been suggested to be the main cause for gene family expansion in plants (Yuan et al., 2015). With that definition, StHsf012 and StHsf013 on chromosome 9 (location: $48.449647 \mathrm{Mb} / 48.466395 \mathrm{Mb}$ ) was found to be a pair of tandem duplications. A chromosome region containing two or more genes within $200 \mathrm{~kb}$ can be defined as a gene cluster (Holub, 2001; Zhang et al., 2015). Therefore, StHsf012 and StHsf013 also belong to a gene cluster.

\section{Phylogenetic Analysis, Exon-Intron Organization and Conserved Motifs of the StHsf Family}

The phylogenetic tree created by the alignment of these fulllength StHsf sequences illustrated the evolution relationships of Hsf members in potato (Figure 3A). These StHsfs are classified into A, B and C classes as described above. The class A members develop into three smaller groups in comparison with class B and class $\mathrm{C}$ Hsfs which respectively form into a single group. The class $\mathrm{C}$ member (StHsf027) has an even closer evolution relationship with class A members than class B members implying that class C Hsf probably evolved from class A Hsfs.

The gene structure diagram depicted that most StHsf genes have one intron, while 3 genes (StHsf012, StHsf013, and StHsf022) contain two introns (Figure 3B). The length of intron exhibited certain degrees of variation, which is similar to that in other plants like Arabidopsis (Nover et al., 2001) and tomato (Baniwal et al., 2004). The exon-intron structure of most homologous gene pairs shared the similarity (StHsf006/StHsfo08, StHsf004/StHsf018, StHsf010/StHsf011, StHsf012/StHsf013, StHsf015/StHsf016); while some homologous genes had several differences in intron numbers (StHsf020/StHsf021), intron length (StHsf022/StHsf023, StHsf024/StHsf025), and intron position (StHsf001/StHsf003).

Twenty conserved motifs of the deduced StHsf proteins were predicted by MEME motif detection software to reveal the conservation and diversification of these proteins in structure and function (Figure 3C). The details of the 20 putative motifs were shown in Table 3. As shown in Figure 3C, motif 1 was connected with motif 2 and motif 4 closely. This joint cluster of motifs 1,2, and 4 represents the most conservative domain (DBD) existing in all 27 StHsf proteins. Besides, motifs 3, 5, and 
TABLE 3 | Analysis and distribution of conserved motifs in potato StHsfs.

\begin{tabular}{|c|c|c|c|}
\hline Motif & $E$-value & Width & Best possible match \\
\hline 1 & $1.8 e-945$ & 50 & FIVWDPPEFARDLLPKYFKHNNFSSFVRQLNTYGFRKIDPDRWEFANEWF \\
\hline 2 & $1.0 \mathrm{e}-360$ & 29 & PPPFLTKTYEMVDDPSTDHIISWNRNGTS \\
\hline 3 & $2.4 \mathrm{e}-218$ & 50 & LMMELVKLRQHQQATDHYMQTMTQRLQATEQRQQQMMSFLAKAMQNPGFV \\
\hline 4 & $1.9 \mathrm{e}-184$ & 20 & LRGQKHLLCNIHRRKTWHSH \\
\hline 5 & $1.7 \mathrm{e}-050$ & 15 & RIGYEEEIERLRRDK \\
\hline 8 & $1.2 \mathrm{e}-013$ & 26 & MLSSELTHMKKLCNDIIYFMSNYVKP \\
\hline 9 & $1.8 \mathrm{e}-014$ & 19 & WWNLKHMHHLTEQMGHLTP \\
\hline 10 & $2.3 e-012$ & 50 & HVGGFSHYIKSEPLEFGEANGFQVSELEALALEMQGFGRARKDQQEEYTI \\
\hline 11 & $1.3 e-011$ & 28 & CVDTLADGQIVRYQPIMHEAAKWINQCI \\
\hline 12 & $5.7 e-007$ & 50 & AGMRQNCSIDLDESISCADSPAISYPQLNVDVGPKASGIDMNSEPNGNTT \\
\hline 16 & $2.8 \mathrm{e}-001$ & 50 & QPHLMSDSGFPFNSCLSIIPEIQYSPTVPGQAKIPQFPEADALNSQADH \\
\hline 17 & 4.6e-001 & 6 & ACVEVG \\
\hline 18 & $7.6 e+000$ & 22 & RSPDANFDKDAFCQSSPSSGTP \\
\hline 19 & $1.3 e+000$ & 20 & KKRKMQWEELEGDNEGRKK \\
\hline 20 & $1 . e+001$ & 8 & MEPHHYYH \\
\hline
\end{tabular}

8 were also conserved domains in StHsfs. Motif 5 and motif 3 were inferred as the OD region of StHsfAs, and motif 5 and motif 8 were considered as the OD of StHsfBs according to the conversed hydrophobic positions of HR-A and HR-B in StHsfs (Supplementary Figure S2). Moreover, StHsf members within the same subgroups were generally found to share a common motif organization. A unique motif, named as motif 6 , was detected in 15 of 18 Hsfs. This motif was close to the $\mathrm{C}$ terminal and was deduced to be an AHA motif. These analyses suggested that most of the closely related members in the phylogenetic tree shared common motifs within the same group and possessed similar exon-intron arrangements.

\section{StHsfs Expression in Different Organs and Tissues}

A StHsf expression heatmap of 12 different organs and tissues, which established using RNA-seq data (Supplementary Table S2), showed a higher expression of most genes in tubers, callus, shoots and roots than in other tissues, such as leaves, stamens and petals (Figure 4). Some StHsf genes shared a highly similar expression profile in various potato tissues. For example, StHsf012 and StHsf013, belonging to $H s f A 7$, were highly expressed in tubers and callus while lowly expressed in stamens, petals, sepals and carpels. Other members of B group, such as StHsf019, StHsf020, StHsf021, StHsf022, StHsf023, and StHsf024, exhibited high levels of expression in vegetative organs, such as shoots, roots, tubers, stolons and callus, suggesting an involvement of class B members in plant vegetative growth. Some members of class A including StHsf0O2, StHsfOO3 and class C member StHsf027, were characterized by high expression amounts in vegetative organs and also in sepals, petals, carpals and the whole flowers than other StHsf members. Within the HsfA1 group, StHsf001 was highly expressed in roots, tubers and carpels while StHsf002 had high expression in tubers, stolons, flowers, petals, sepals and callus and the expression of StHsfOO3 were high in roots, flowers, carpels and sepals. This analysis indicated that the plants have adaptation reaction to harmful environment through compensation of Hsf genes to lighten the menace action of adversity.

\section{Promoter and qRT-PCR Analyses of StHsf Genes on Stress Responses}

Response elements of StHsf genes' promoters, including Heat Stress Element (HSE), C-Repeat Binding Factor (CBF), ABA Responsive Element (ABRE), Dehydration-Responsive Element (DRE), and Low Temperature Responsive Element (LTRE) were displayed in Figure 5. The promoter analyses showed that nearly all of these genes had multiple HSEs, CBFs, ABREs, DREs, and LTREs in their promoter regions except StHsf024 whose promoter sequence has not been completed. All these genes had multiple DREs suggesting that they are responsive to drought stress. Some genes are lacking of one or two response elements, such as StHsf007 without HSE and StHsfOOs without LTRE, which implied that the expression of these genes might be weakly influenced by the corresponding stresses or not be directly induced by these stresses. In general, the promoter analysis suggested that all of StHsf genes should be responsive to various abiotic stresses.

To validate this hypothesis, qRT-PCR was used to determine the expression profiles of StHsf001, 004, 005, 007, 008, 009, 012, $014,015,016,017,018,019,021,022,024,026$, and 027 genes in leaves of potato plants subjected to heat, drought and cold 


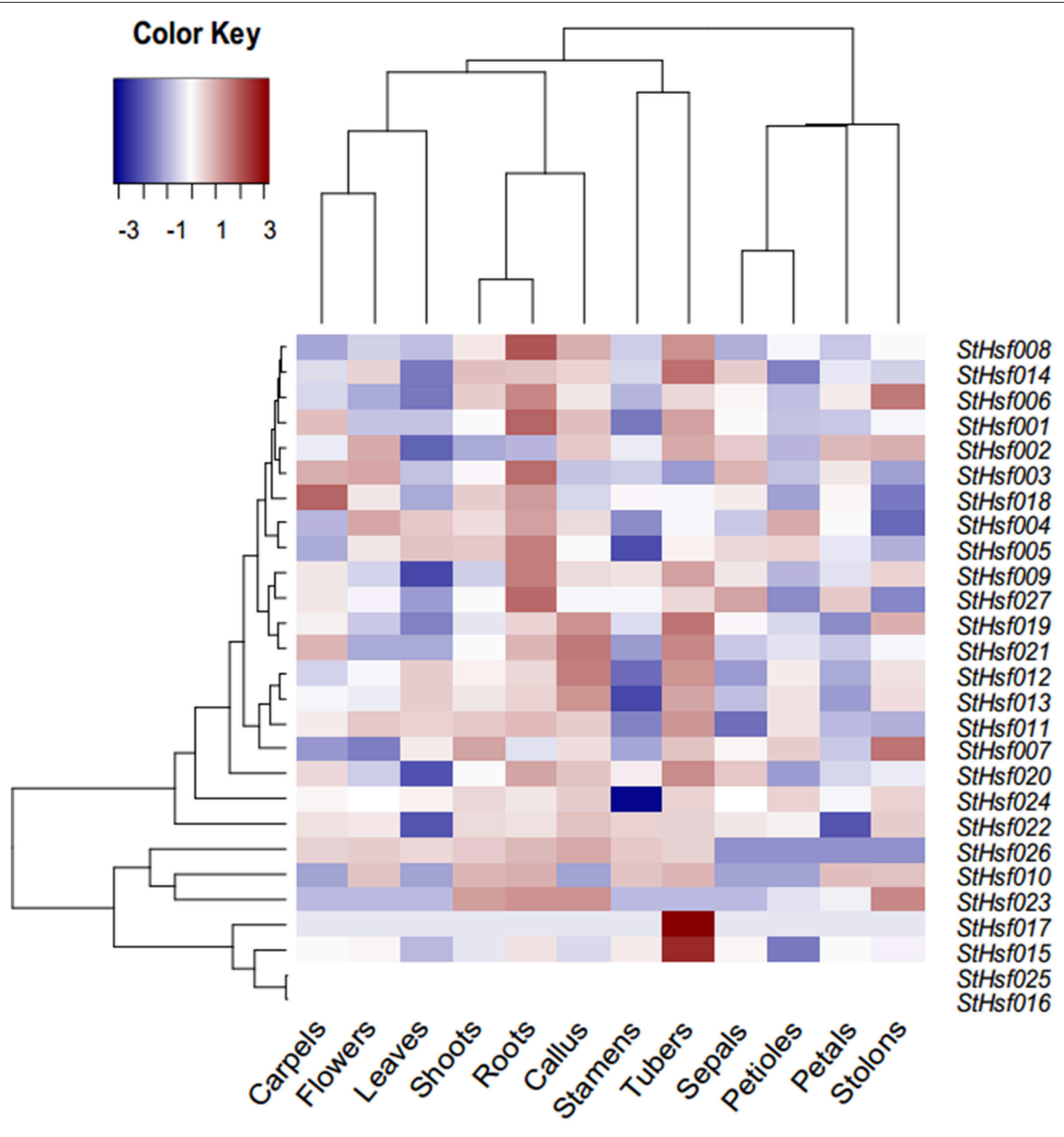

FIGURE 4 | Expression profiles of the StHsf genes in different potato organs and tissues.

stresses (Figure 6). These StHsf genes were selected from all subgroups and the primers were presented in Supplementary Table S1. The heat, drought and cold responsiveness of these genes was examined in the leaves of 1-month-old plants with short- $(2 \mathrm{~h}$ and $6 \mathrm{~h})$ and long-term $(24 \mathrm{~h})$ stress treatments. In order to clarify which genes play the major role during different stress conditions as a whole, the expression of StHsf001 at $0 \mathrm{~h}$ under the corresponding stress was set to 1 and the expression of other genes in different stages of treatment were compared with that. Generally, the expression levels of StHsf004, StHsfoO5, StHsf007, StHsf009, and StHsf014 were all higher than other genes during different stresses. Specifically, StHsf004, StHsf005, and $S t H s f 009$ became predominant $S t H s f$ transcripts during heat stress, especially StHsf004 and StHsf005 whose expression levels were approximately 200 300 times higher than that of the other members (Figure 6A); while StHsf004, StHsf007, StHsf009, and $S t H s f 014$ all played a leading role under drought stress (Figure 6B) and cold stress (Figure 6C). In addition, constitutive expression of StHsf004, StHsf005, StHsf007, and StHsf012 was observed before stress treatments, which is in accordance with the expression heatmap of the StHsf genes in potato leaves (Figure 4).

This qRT-PCR data was also used to analyze the expression changes of each gene in heat, drought and cold stress treatments separately (Supplementary Figure S3). In general, the expression amounts of selected genes in each stress treatment had fluctuated over these $24 \mathrm{~h}$. The expression levels of most genes began to show dramatic changes at 2 or $6 \mathrm{~h}$, which indicated that StHsfs are sensitive to abiotic stresses. In particular, during heat stress, the expression of most class A members in leaves was up-regulated. For instance, the transcript levels of StHsf001, StHsf004, StHsf007, StHsf016, and StHsf017 were up-regulated and reached the peak level at $2 \mathrm{~h}$, and the expression of StHsf005, StHsf008, StHsf009, StHsf014, StHsf015, and StHsf018 was peaked at 6 or $24 \mathrm{~h}$. However, the mRNA level of StHsf012 was down-regulated with a $2 \mathrm{~h}$ heat treatment but an increase was observed in the $6 \mathrm{~h}$ treatment. Heat up-regulation was also observed in most $H s f B$ members with $2 \mathrm{~h}$ treatment, such as StHsf022, StHsf024, and StHsf026, especially StHsf022 whose expression amount at $2 \mathrm{~h}$ soared to about 50 -fold of expression level at $0 \mathrm{~h}$. Conversely, the 


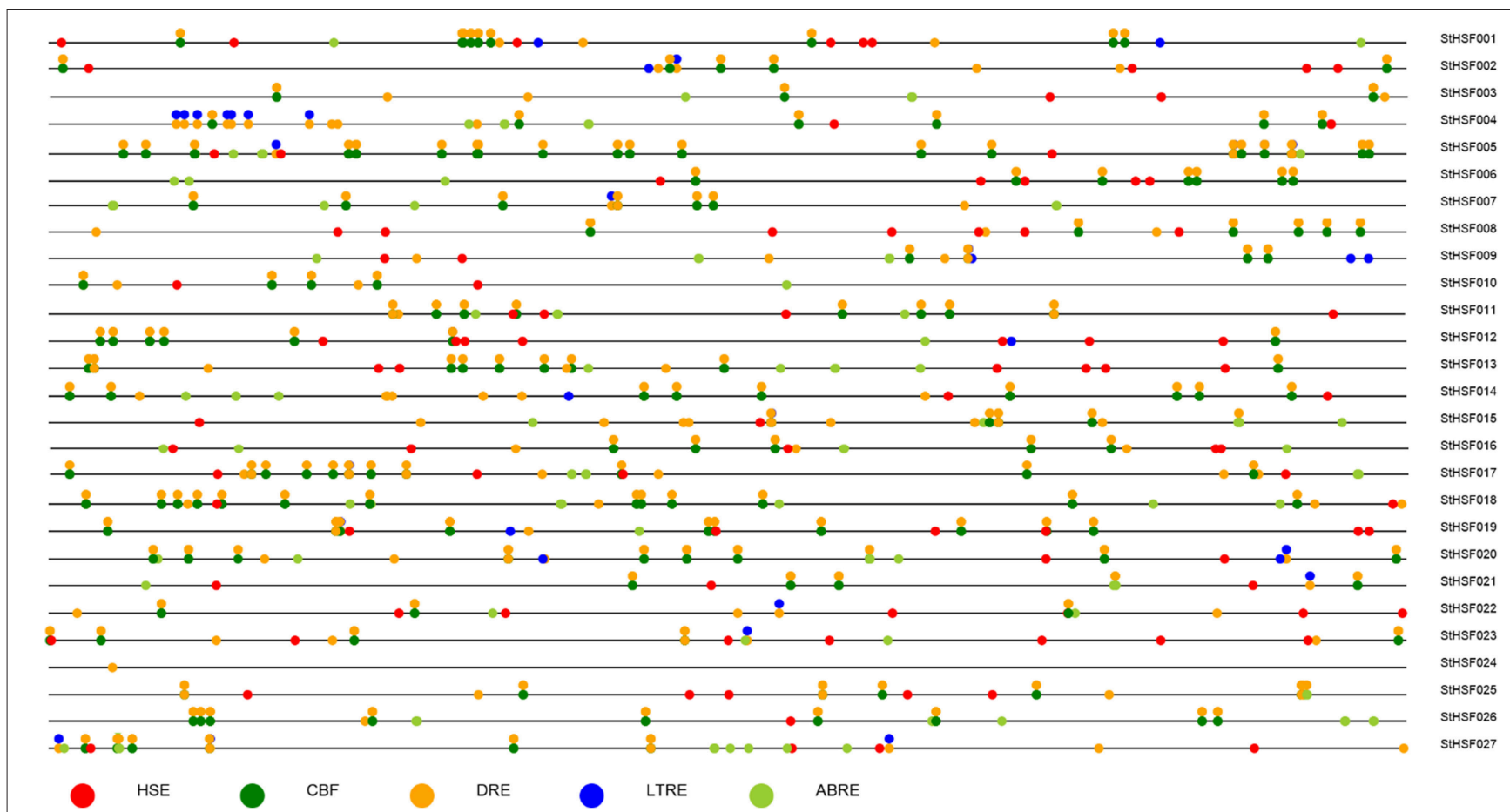

FIGURE $\mathbf{5}$ | Promoter analysis of $\mathbf{2 7}$ deduced StHsfs. The circles in different colors represent different stress response elements of the $-2 \mathrm{~Kb} 5^{\prime \prime}$ upstream region of 27 StHsfs.

gene expression of StHsfo19 declined gradually and dramatically under heat stress. The expression level of StHsf027, the class $\mathrm{C}$ member, declined in the $2 \mathrm{~h}$ treatment but increased at $6 \mathrm{~h}$ treatment.

Although the major role of Hsfs is well known to be the regulation of heat-responsive genes involved in heat acclimatization, it was also of interest to detect whether this family is involved in response to other major abiotic stresses such as drought and cold stresses. The expression of these genes was induced by drought stress as the same as heat stress but the expression pattern of each gene was different from that in heat condition. More exactly, the expression levels of most of these genes mediated by drought stress witnessed a downward trend from 0 to $2 \mathrm{~h}$ onwards, which was in contrast to the expression pattern under heat treatment. Some genes such as StHsf005 and StHsf027, however, were up-regulated by drought transiently. As for cold stress, these genes presented a different expression pattern from heat and drought. Apart from StHsf007, StHsf009, StHsf014, StHsf018, and StHsf022, whose expression amounts had an increase trend, the other selected genes dipped steadily.

\section{Construction of Co-expression Network and Identification of StHsf-Related Genes}

In order to delve into the potential regulatory relationship between $S t H s f s$ and their related genes, a co-expression network was constructed using WGCNA according to the RNAseq data. As shown in Figure 7, 15 modules containing different StHsfs and their co-expressed genes were formed based on the correlation of biological function among genes. Some StHsfs
(StHsf002, StHsf003, StHsf006, StHsf014, StHsf015, StHsf016, StHsf017, StHsf018, and StHsf025) were not shown in Figure 7 because of their low expression levels in RNAseq data. Module 1, which contained StHsf005 and other 642 related gene members, was the largest module among the 15 modules; while module 15, containing only 10 genes, was the smallest module (Figure 7). Because module 1 contained more genes than other modules, we hypothesize that module 1 is involved in more biological functions than other modules.

The co-expression network of Hsfs and their related genes in potato was created by Cytoscape with TOM (Topological overlap matrix) value 0.07 (Figure 8). The genes in a module were formed into a circle with StHsf as a center (red points) and other related genes around (blue points). The sequence numbers and potential functions of these genes related to StHsfs in Spud DB Potato Genomics Resources were provided in Supplementary Table S3. Proteins, encoded by these genes, are not only StHsps like Heat shock cognate $70 \mathrm{kDa}$ protein (PGSC0003DMT400001180) and $10 \mathrm{kDa}$ chaperonin (PGSC0003DMT400060280), but also other genes with a variety of functions, such as DELLA protein (PGSC0003DMT4000 49445), MAPK (PGSC0003DMT400077272) and MAPKKK (PGSC0003DMT400057171) in the module 3 which contained StHsf007. Except the relationship between StHsfs and their correlated genes, there also existed direct or indirect interactions between StHsfs, such as StHsf001, StHsf005, StHsf008, StHsf009,

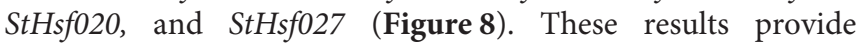
some basis for further study of function mechanism of StHsfs. 


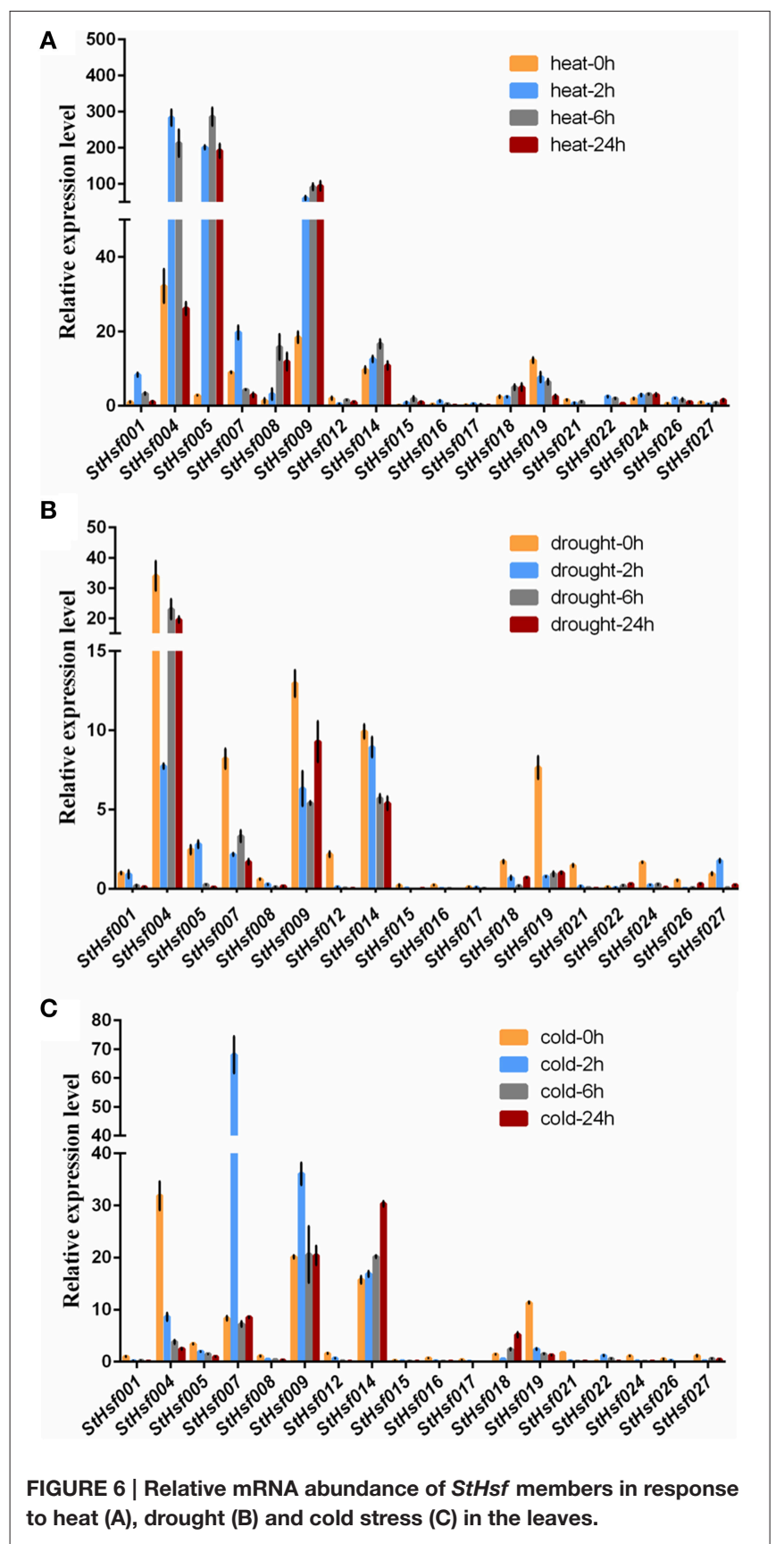

\section{DISCUSSION}

\section{Hsfs in the Same Class and Subclass Share Similar Gene Structures, Conserved Motifs and Regulatory Functions}

Hsfs, which exist extensively in all plant species, have been considered as a sort of important regulators in response to abiotic stresses based on the researches of Hsfs in some species like Arabidopsis (Nover et al., 2001), tomato (Mishra et al., 2002), apple (Giorno et al., 2012), and cabbage (Ma et al., 2014). Nevertheless, few researches on Hsfs in potato have been reported. As the potato genome sequence has been completed, 27 Hsf genes from Solanum tuberosum were identified and analyzed in phylogenetic relationships, gene structures, conserved motifs, chromosomal locations, promoters and expression patterns. These Hsf members which belong to the same class and subclass had a close evolutionary relationship and shared a similar exonintron structure and motif composition, implying their similar regulatory functions.

Orthologous genes which exist in different species from a common ancestor may or may not have the same function; while paralogous genes within a single species that created by gene duplication may evolve new functions related to the original genes (Thornton and DeSalle, 2000; Guo et al., 2008). Therefore, gene orthology analysis can be used as a preliminary method to explore the function of candidate genes (Wang et al., 2014). HsfAla has been reported as an irreplaceable master transcription factor of induced heat tolerance in tomato but no similar Hsfs were found in Arabidopsis (Lohmann et al., 2004; Von Koskull-Döring et al., 2007). In potato, HsfAla was not identified according to Figure 1. Therefore, further function analysis of HsfA1 in potato is required to justify whether there exists a master regulator as in tomato. HsfB1, which was considered to enhance the activity of recruiting histone acetyltransferase (HAC1) by collaborating with tomato HsfAla, has similar functions in tobacco and soybean but acts as a repressor of HsfAs in Arabidopsis (Von Koskull-Döring et al., 2007). Further investigation of the HsfB1 sequences of tomato, Arabidopsis and potato exposed that the histone-like motif (GRGKMMK) in tomato $\mathrm{HsfBl}$ is also found in potato but is changed by GSRMTETK in Arabidopsis, indicating that potato HsfB1 probably has a similar function as tomato $\mathrm{HsfB} 1$ (Supplementary S1; Bharti et al., 2004; Von Koskull-Döring et al., 2007; Zhu et al., 2012).

From the paralogous genes analysis of potato, class A Hsf members were observed to develop into several smaller groups whereas class B and class C members just formed a single group respectively, suggesting that the class A Hsfs might be derived from different gene ancestors compared with class B and class C Hsfs. Therefore, the class A was reported as a paraphyletic group, while the class $\mathrm{B}$ and class $\mathrm{C}$ were regarded as a monophyletic group (Scharf et al., 2012). Gene duplication and gene deficiency events usually occurred at different stage of plants evolution resulting in Hsfs diversity (Lin et al., 2006). One pair of paralogous StHsf genes, StHsf012/StHsf013, appeared to have undergone tandem duplication, which suggested that they probably derived from a same gene ancestor and have the same function. StHsf015, StHsf016, and StHsf017, which are unique genes in potato, might have mutation in the process of evolution. Closer observation of sequences in DBD and insertion area between HR-A and HR-B reveals that mutation such as replacement or deficiency of some amino acids may cause changes of their structure and function. For example, the $\alpha_{3}$ conserved sequence -RQLN- was replace by -CQLN-, - YQLN-, and - YQLN- in StHsf015, StHsf016 and StHsf017, respectively (Supplementary Figure S1). To our knowledge, $\mathrm{R}$ is a kind of positively charged amino acid while $\mathrm{C}$ and $\mathrm{Y}$ are polar amino acids without charge, which might have an impact on 


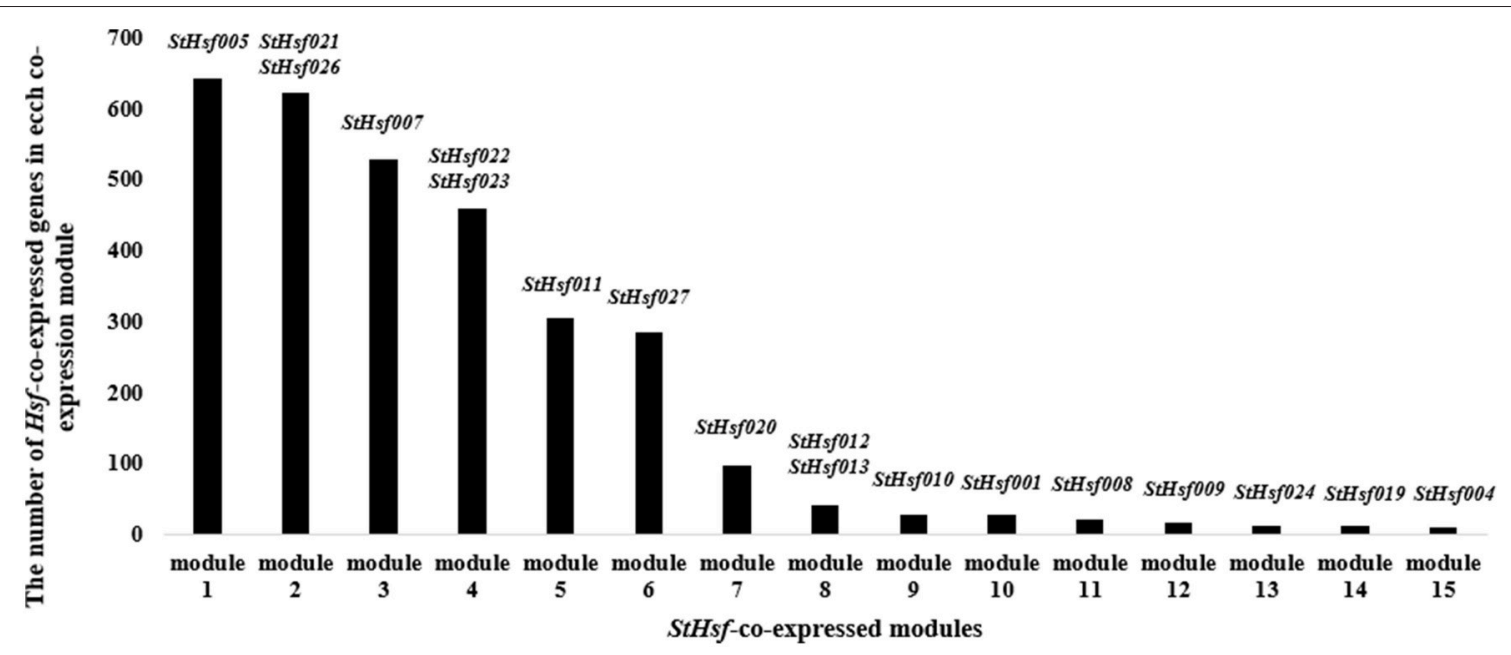

FIGURE 7 | The number of StHsf-co-expressed genes in each co-expression module. The modules are numbered according to the gene numbers. StHsf genes involved in the corresponding module are placed at the top of each column.

their function. Also, the OD regions of StHsf015, StHsf016, and StHsf017 were found to be incomplete compared with the normal OD domain, which offered another possibility of their changed function (Figure 3C and Supplementary Figure S2).

The number of potato Hsf members is close to plant species like Arabidopsis, tomato, rice, maize, poplar, and pepper in comparison with soybean. This probably results from the double duplications of genome in soybean but only a single replication in other plants during evolution (Blanc et al., 2000; Schlueter et al., 2004). Several Hsfs are specific to monocots or dicots. For example, the Hsf members of A9, B3, and B5 are restricted to dicots, and $\mathrm{C} 2$ are characteristic of monocots, suggesting the evolution of these subclasses after the divergence of monocots and dicots (Li et al., 2014).

Transcriptional activity of HsfA normally depends on the AHA motif in the C-terminal (Bharti et al., 2000). However, not all HsfA members contain this motif like StHsf005 (HsfA3) (Figure 3C). It was confirmed that HsfAs without an AHA motif could be activated by forming hetero-polymers with other HsfAs (Guo et al., 2008; Li et al., 2014). Most of the class B and C Hsfs do not possess the transcription activation ability like class A Hsfs due to the deficiency of AHA motif in their CTADs. Instead, the class B Hsf members are characterized with a tetrapeptide -LFGV- in their C-terminal region, which is proposed to act as a repressor motif in the transcription machinery (Ikeda and Ohme-Takagi, 2009). This tetrapeptide can be found in StHsf019, StHsf021, StHsf022, StHsf023, and StHsf024 (Supplementary S1), indicating the probable repression role of these five Hsfs in potato.

\section{Hsf Members Play Crucial Roles in Potato Growth and Development and in Response to Intricate Abiotic Stresses}

According to Hsf genes discovered so far, in-depth researches and new technologies enable the identification of more $H s f$ genes in various organisms. Considering the important roles that Hsfs play during plant development and in response to various stresses, it is not surprising that we identified so many Hsf family members in potato. The present research confirmed the involvement of Hsfs in tolerance to various stresses in potato, which is in agreement of the previous studies of Hsfs in Arabidopsis, rice, tomato and any other species (Mishra et al., 2002; Guo et al., 2008; Chauhan et al., 2011; Hahn et al., 2011; Yoshida et al., 2011).

Expression patterns analysis of all predicted Hsf members in 12 different potato organs or tissues uncovered that StHsf genes in the same group have a similar expression profile in potato tissues, implying that they may participate in a similar developmental process or regulatory pathway. Take the expression of StHsf012 and StHsf013 as an example, they were lowly expressed in the organs related to flower, suggesting that they may not participate in the regulation of flower development under non-stress condition. HsfA9, which has been reported in Arabidopsis to specifically express in seeds (Von Koskull-Döring et al., 2007), also expressed in carpels, roots and shoots of potato. This results indicated that Hsf members have different expression patterns in different plant species. All these Hsfs perform their own functions in different organs and tissues to make sure the normal growth and development of plants. Although some Hsfs expressed lowly in certain tissues, this does not mean that they are non-functional in these tissues. The expression of some Hsfs need to be induced by different stresses.

The constitutively expressed members (e.g. StHsfo04, StHsf007, and StHsf012) expressed at relatively higher levels (Figure 6), which fits with the expression results of StHsfs in leaves as shown in Figure 4. In tomato, HsfA1 was found to be a constitutively expressed gene and $H s f A 2$ was an induced up-regulated gene (Nishizawa et al., 2006; Von KoskullDöring et al., 2007). In potato, however, the expression of $H s f A 1 b$ (StHsf001) induced by heat stress treatment and HsfA2 (StHsf004) constitutively expressed under non-stress condition 


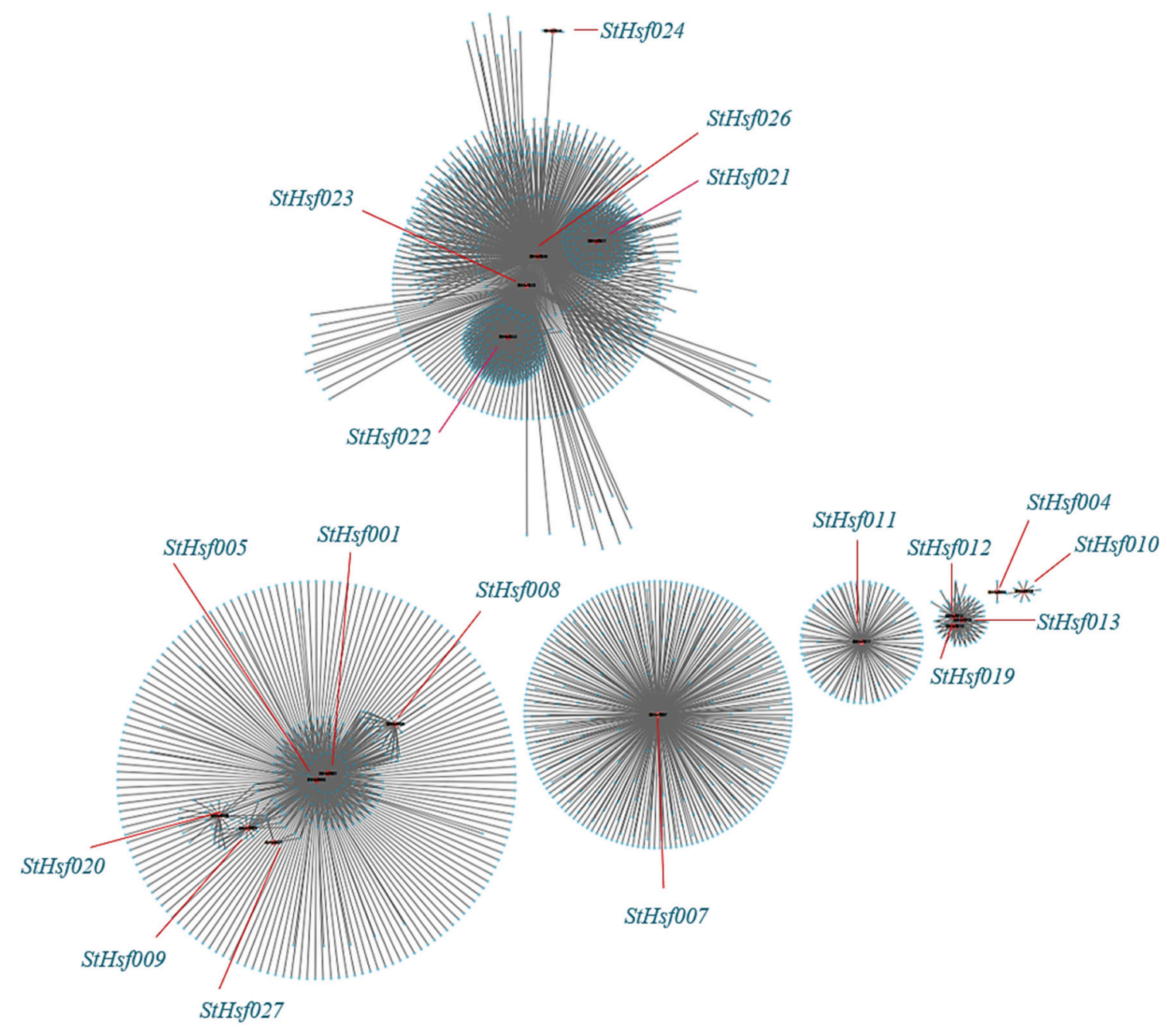

FIGURE 8 | Co-expression network of $\boldsymbol{H s f s}$ and their co-expressed genes in potato. TOM (Topological overlap matrix) value is 0.07 . Nodes represent StHsf and co-expressed genes in potato, edges indicate pairwise correlation constructed by WGCNA. Red circles indicate Hsfs, blue circles represent Hsps. The network was created using Cytoscape (see Materials and Methods). The length of the line segment doesn't stand for the closeness of the relationship between StHsf and each gene; it is simply for distinguishing different genes.

and up-regulated by heat stress (Figure 6), which is opposite to the situation in tomato. The results of Liu and Charng (2013) have demonstrated that the function of HsfAls can be replaced by HsfA2 in the absence of HsfA1s because of their high degree of sequence homology. But whether HsfA2 could perform the similar functions as HsfA1s in the low expression of HsfA1s needs further study. StHsf004, StHsf005, and StHsf009, belonging to A2, A3, A5 group, were the main StHsfA members that were up-regulated at a very high level during heat stress. Other class A genes that were up-regulated by heat were A4 and A8 group members (StHsf007, StHsf008, and StHsf014), but they were expressed at a low level under heat stress, compared with the A2, A3, and A5 groups. In terms of class B of the StHsf family, not all $S t H s f B$ members were down-regulated by heat; actually, the expression levels of the StHsf022, StHsf024, and StHsf026, which are B3, B4 and B5 members, increased after an initial induction. In class C, StHsf027 was constitutively expressed in most organs. In leaves, the expression level of the $\mathrm{C}$ group was even higher than those of the A1 group and was up-regulated after a temporary descent with heat stress treatment.

The promoter analysis showed that most StHsfs contain multiple response elements in their promoter regions (Figure 5), presumably suggesting that these StHsfs could be transcriptionally activated by the combination of response elements and some stress-induced tans-acting factors. For example, Chen et al. (2010) has demonstrated that DREB2C can trans-activate the DRE-dependent transcription of HsfA3 by preferentially binding to the distal DRE2 located in the HsfA3 promoter under heat stress. StHsf007, which is lacking of HSE in its promoter, had an up-regulated expression in heat treatment (Figure 6A). One possibility is that under heat stress, some trans-acting factors like DREB2C might combine with the DRE located in the StHsf007 promoter to activate the expression of $\mathrm{StH}$ sf007. However, the gene expression is a complex biological 
process and not only modulated by transcriptional regulation which is also a complicated process. The exact mechanism of gene expression needs further research.

The promoter analysis of StHsfs and a number of studies about $H s f$ gene family have provided evidence of their function to other abiotic stresses, reflecting that Hsfs are crucial regulators in the regulation of various stress responses (Swindell et al., 2007; Hu et al., 2009; Zhang et al., 2015). In respect to the function of HsfA3, it was reported to be induced by drought stress and was considered as a drought signal molecular in Arabidopsis (Sakuma et al., 2006; Von Koskull-Döring et al., 2007); while there is no considerable change of the expression of StHsfA3 (StHsfo05) after drought treatment in potato, which is similar to HsfA3 in tomato (Bharti et al., 2000). The up-regulation of StHsf018 and StHsf022 was also seen in the leaves of cold-stressed plants as well as StHsf007, StHsf009, and StHsf014. These data indicated that some of StHsf members may have a regulatory role in potato adaptation to heat, drought and cold stresses. These genes showed different expression patterns during different stresses, implying that each StHsf may function differently by reacting with different downstream stress protection genes. It is also known that environmental stresses can induce epigenetic and somatic genome variations ( $\mathrm{Li}, 2016)$ and that Hsfs are among the earliest regulators to stresses, as indicated in the present study. It is interesting to investigate whether Hsfs play a role in directly or indirectly regulating epigenetic and somagenetic variations.

\section{The Co-expression Network between Potato Hsfs and Their Correlated Genes Help to Excavate the Regulatory Mechanisms of Stress Responses}

Hsfs have been demonstrated to play key roles in the tolerance to various adverse environment by reacting with different genes especially Hsps (Swindell et al., 2007; Hu et al., 2009). The expression of the same Hsfs can induce the expression of different groups of Hsps and the different Hsfs can transcriptionally activate the expression of the same sets of Hsps under different stresses, which indicated that there exists a complex regulatory network between $H s f s$ and Hsps. For example, in Arabidopsis, HsfA3 has been confirmed to be in control of the expression of $H s p 70 b, H s p 19.9-P$, and Hsp22.0-ER under heat treatment while another researches have reported that Hsp18.1-CI, Hsp26.5-MII, and $H s p 70$ were transcriptionally regulated by HsfA3 in response to drought stress (Sakuma et al., 2006; Yoshida et al., 2008). In this study, Hsf005 (HsfA3) and Hsf007 (HsfA4b) were found to regulate the expression of $H s p 17$ members, which suggested that some Hsps express under the control of different Hsfs and involve in diverse regulatory pathways (Supplementary Table S3). However, the understanding of precise regulatory mechanisms among Hsfs and Hsps during abiotic stress responses is not clear.

Besides Hsps, other genes with different functions are also related to $H s f s$ and induced by various abiotic stresses. Some genes are expressed specifically in response to the distinct stresses and have no significant response to other stresses, while another genes play important roles in the crosstalk of multiple adverse stresses. For example, genes like DREB1a and MAP65 were affected notably by heat stress but did not have striking expression amounts under drought or salt stress ( $\mathrm{Hu}$ et al., 2009). However, Myb-like DBD protein and CBL-interacting serine/threonine-protein kinase, which were demonstrated to participate in the signal pathways in the tolerance to drought and salt stresses in the previous studies, have also been confirmed to be functional in heat stress (Hu et al., 2009). The potential reason is that various abiotic stresses can trigger the same response mechanisms such as change of membrane permeability (Tsvetkova et al., 2002), production of ROS (reactive oxygen species) (Miller and Mittler, 2006), and consequently generate a lot of protective genes to resist the adverse stresses.

Co-expression analysis, as an important approach to find the new genes and explore the genes potential functions, has already been applied in discovering the genes taking part in the specific biological processes in Arabidopsis, maize, and Populus (Higashi and Saito, 2013). The co-expression network between StHsfs and their co-expressed genes provided some information and direction to excavate the regulatory mechanisms between $H s f s$ and $H s f$-associated genes during development and stress responses and can be confirmed by testing the expression changes of detected genes through overexpression or knockout of the corresponding $H s f s$. Many Hsps like sHsp-CI (PGSC0003DMT400007587), Hsp70 (PGSC0003DMT400008407), Hsp83 (PGSC0003DMT4000 14217) in module 1 were co-expressed with StHsf005 (HsfA3) (Supplementary Table S3) indicating that Hsf005 plays a significant role in heat resistance (Figure 6A). Another important result that the expression of StHsf007 ( HsfA4b) increased markedly under short-time cold stress treatment can be deduced that some genes with the function to respond to cold stress may be induced by $\mathrm{Hsf007}$ (Figure 6C). DELLA proteins, which have been reported to contribute to the increase in freezing tolerance and cold acclimation (Achard et al., 2008; Lee and Thomashow, 2012), can be detected to co-express with $\mathrm{Hsf007}$ in module 3. This evidence primarily proves our hypothesis and further experiments are necessary to investigate the mechanisms of Hsf007 response to cold stress. Furthermore, in this study, we find MAPK and MAPKKK (Supplementary Table S3) among the co-expressed genes of StHsf007. MAPKs (Mitogen-activated protein kinases) are protein Ser/Thr kinases that transform extracellular stimuli to a wide range of cellular responses (Cargnello and Roux, 2012). The MAPK pathway has the highest number of top-ranked proteins among signaling pathways (Du et al., 2012) and is the pathway heavily involved in plant tolerances to stresses ( $\mathrm{Li}$ et al., 2016). The co-involvement of Hsf007 with the signaling MAPK pathway proteins in the same module suggests that Hsfo07 likely plays a role in the signaling in response to some abiotic stresses. Therefore, Hsfoo7 is among the key candidate genes of which the molecular mechanisms in stress tolerance should be further illustrated.

\section{CONCLUSIONS}

In summary, a total of $27 \mathrm{StHsfs}$ in the Solanum tuberosum genome were identified. A series of analyses of these genes, including phylogeny, chromosomal location, gene structure, expression profiling and abiotic stress responses, was performed 
by bioinformatics and qRT-PCR methods. The StHsfs were unevenly distributed in 10 of the 12 chromosomes. The amino acids numbers inserted in HR-A/B domain are based to categorize $27 \mathrm{StHsfs}$ into three large classes. In the phylogenetic tree, the majority of subfamilies contained members from potato, tomato, cucumber, soybean and Arabidopsis, which suggested that the structures and functions of most Hsfs were conserved during evolution. A majority of StHsfs were found expressed in more than one tissue in potato according to the RNAseq data analysis. The promoter analysis suggested that nearly all StHsfs were activated in response to diverse abiotic stresses. An extensive expression analysis indicated that StHsf genes may play various roles in different biological processes in plants: StHsf004, StHsf005, StHsf009 become predominant StHsfs during heat stress; StHsf004, StHsf007, StHsf009, and StHsfol4 function as predominant genes under both drought and cold stresses. Furthermore, the co-expression network implied that there is a complex transcriptional regulatory network between StHsfs and their correlated genes. Besides, the co-expression network provides some information and direction to excavate the regulatory mechanisms between $H s f s$ and their co-expressed genes during development and stress responses, and also helps to select candidate Hsf genes, such as StHsfO05 and StHsf007, for future functional analyses.

\section{REFERENCES}

Achard, P., Gong, F., Cheminant, S., Alioua, M., Hedden, P., and Genschik, P. (2008). The cold-inducible CBF1 factor-dependent signaling pathway modulates the accumulation of the growth-repressing DELLA proteins via its effect on gibberellin metabolism. Plant Cell 20, 2117-2129. doi: 10.1105/tpc.108.058941

Ahuja, I., de Vos, R. C. H., Bones, A. M., and Hall, R. D. (2010). Plant molecular stress responses face climate change. Trends Plant Sci. 15, 664-674. doi: 10.1016/j.tplants.2010.08.002

Akerfelt, M., Morimoto, R. I., and Sistonen, L. (2010). Heat shock factors: integrators of cell stress, development and lifespan. Nat. Rev. Mol. Cell Biol. 11, 545-555. doi: 10.1038/nrm2938

Bailey, T. L., Boden, M., Buske, F. A., Frith, M., Grant, C. E., Clementi, L., et al. (2009). MEME SUITE: tools for motif discovery and searching. Nucleic Acids Res. 37, W202-W208. doi: 10.1093/nar/gkp335

Baniwal, S. K., Bharti, K., Chan, K. Y., Fauth, M., Ganguli, A., Kotak, S., et al. (2004). Heat stress response in plants: a complex game with chaperones and more than twenty heat stress transcription factors. J. Biosci. 29, 471-487. doi: 10.1007/BF02712120

Bharti, K., Schmidt, E., Lyck, R., Heerklotz, D., Bublak, D., and Scharf, K. D. (2000). Isolation and characterization of HsfA3, a new heat stress transcription factor of Lycopersicon peruvianum. Plant J. 22, 335-365. doi: 10.1046/j.1365313x.2000.00746.x

Bharti, K., Von Koskull-Döring, P., Bharti, S., Kumar, P., Tintschl-Körbitzer, A., Treuter, E., et al. (2004). Tomato heat stress transcription factor HsfB1 represents a novel type of general transcription coactivator with a histone-like motif interacting with the plant CREB binding protein ortholog HAC1. Plant Cell 16, 1521-1535. doi: 10.1105/tpc.019927

Blanc, G., Barakat, A., Guyot, R., Cooke, R., and Delseny, M. (2000). Extensive duplication and reshuffling in the Arabidopsis genome. Plant Cell 12, 1093-1101. doi: 10.1105/tpc.12.7.1093

Cargnello, M., and Roux, P. P. (2012). Activation and function of the MAPKs and their substrates, the MAPK-activated protein kinases. Microbiol. Mol. Biol. Rev. 76, 496. doi: 10.1128/MMBR.00013-12

\section{AUTHOR CONTRIBUTIONS}

RT, QY, and WZ conceived and designed the research. RT, WZ, and XS performed qRT-PCR analysis. RT and XL performed the bioinformatics analysis and construction of co-expression network. RT, XS, XL, JC, and MW analyzed the data. RT and QY wrote the manuscript.

\section{ACKNOWLEDGMENTS}

The authors thank Xiu-Qing Li for substantial help in improving the paper. This research was supported financially by grants from the National Pear Industry Technology System (CARS-29), the National Natural Science Foundation of China (11171155), the Priority Academic Program Development of Jiangsu Higher Education Institutions: Modern Horticultural Science (PAPD) and the Research Innovation Project of Jiangsu Province for University Graduate Students (KYZZ15_0181).

\section{SUPPLEMENTARY MATERIAL}

The Supplementary Material for this article can be found online at: http://journal.frontiersin.org/article/10.3389/fpls.2016. 00490

Chauhan, H., Khurana, N., Agarwal, P., and Khurana, P. (2011). Heat shock factors in rice (Oryza sativa L.): genome-wide expression analysis during reproductive development and abiotic stress. Mol. Genet. Genomics 286, 171-187. doi: 10.1007/s00438-011-0638-8

Chen, H., Hwang, J. E., Lim, C. J., Kim, D. Y., Lee, S. Y., and Lim, C. O. (2010). Arabidopsis DREB2C functions as a transcriptional activator of HsfA3 during the heat stress response. Biochem. Biophys. Res. Commun. 401, 238-244. doi: 10.1016/j.bbrc.2010.09.038

Chung, E., Kim, K. M., and Lee, J. H. (2013). Genome-wide analysis and molecular characterization of heat shock transcription factor family in Glycine max. J. Genet. Genomics 40, 127-135. doi: 10.1016/j.jgg.2012.12.002

Czarnecka-Verner, E., Pan, S., Salem, T., and Gurley, W. B. (2004). Plant class B HSFs inhibit transcription and exhibit affinity for TFIIB and TBP. Plant Mol. Biol. 56, 57-75. doi: 10.1007/s11103-004-2307-3

Damberger, F. F., Pelton, J. G., Harrison, C. J., Nelson, H., and Wemmer, D. E. (1994). Solution structure of the DNA-binding domain of the heat shock transcription factor determined by multidimensional heteronuclear magnetic resonance spectroscopy. Protein Sci. 3, 1806-1821. doi: 10.1002 /pro. 5560031020

Döring, P., Treuter, E., Kistner, C., Lyck, R., Chen, A., and Nover, L. (2000). The role of AHA motifs in the activator function of tomato heat stress transcription factors HsfA1 and HsfA2. Plant Cell 12, 265-278. doi: 10.1105/tpc.12. 2.265

Downs, G. S., Bi, Y. M., Colasanti, J., Wu, W., Chen, X., Zhu, T., et al. (2013). A developmental transcriptional network for maize defines coexpression modules. Plant Physiol. 161, 1830-1843. doi: 10.1104/pp.112.213231

Du, D., Lee, C. F., and Li, X.-Q. (2012). Systematic differences in signal emitting and receiving revealed by PageRank analysis of a human protein interactome. PLoS ONE 7:e44872. doi: 10.1371/journal.pone.0044872

Giorno, F., Guerriero, G., Baric, S., and Mariani, C. (2012). Heat shock transcriptional factors in Malus domestica: identification, classification and expression analysis. BMC Genomics 13:639. doi: 10.1186/1471-2164-13-639

Guo, A. Y., Zhu, Q. H., Chen, X., and Luo, J. C. (2007). GSDS: a gene structure display server. Yi Chuan (Article in Chinese) 29, 1023-1026. doi: 10.1360/yc007-1023 
Guo, J. K., Wu, J., Ji, Q., Wang, C., Luo, L., Yuan, Y., et al. (2008). Genome-wide analysis of heat shock transcription factor families in rice and Arabidopsis. J. Genet. Genomics 35, 105-118. doi: 10.1016/S1673-8527(08) 60016-8

Guo, M., Lu, J. P., Zhai, Y. F., Chai, W. G., Gong, Z. H., and Lu, M. H. (2015). Genome-wide analysis, expression profile of heat shock factor gene family (CaHsfs) and characterisation of CaHsfA2 in pepper (Capsicum annuum L.) BMC Plant Biol. 15:151. doi: 10.1186/s12870-015-0512-7

Hahn, A., Bublak, D., Schleiff, E., and Scharf, K.-D. (2011). Crosstalk between Hsp90 and Hsp70 chaperones and heat stress transcription factors in tomato. Plant Cell 23, 741-755. doi: 10.1105/tpc.110.076018

Hartl, F. U., and Hayer-Hartl, M. (2002). Molecular chaperones in the cytosol: from nascent chain to folded protein. Science 295, 1852-1858. doi: $10.1126 /$ science. 1068408

Higashi, Y., and Saito, K. (2013). Network analysis for gene discovery in plant-specialized metabolism. Plant Cell Environ. 36, 1597-1606. doi: 10.1111/pce. 12069

Higo, K., Ugawa, Y., Iwamoto, M., and Korenaga, T. (1999). Plant cis-acting regulatory DNA elements (PLACE) database. Nucleic Acids Res. 27, 297-300. doi: 10.1093/nar/27.1.297

Holub, E. B. (2001). The arms race is ancient history in Arabidopsis, the wildflower. Nat. Rev. Genet. 2, 516-527. doi: 10.1038/35080508

$\mathrm{Hu}$, W. H., Hu, G. C., and Han, B. (2009). Genome-wide survey and expression profiling of heat shock proteins and heat shock factors revealed overlapped and stress specific response under abiotic stresses in rice. Plant Sci. 176, 583-590. doi: 10.1016/j.plantsci.2009.01.016

Ikeda, M., and Ohme-Takagi, M. (2009). A novel group of transcriptional repressors in Arabidopsis. Plant Cell Physiol. 50, 970-975. doi: $10.1093 / \mathrm{pcp} / \mathrm{pcp} 048$

Katagiri, F. (2004). A global view of defense gene expression regulation-a highly interconnected signaling network. Curr. Opin. Plant Biol. 7, 506-511. doi: 10.1016/j.pbi.2004.07.013

Kilian, J., Whitehead, D., Horak, J., Wanke, D., Weinl, S., Batistic, O., et al. (2007). The AtGenExpress global stress expression data set: protocols, evaluation and model data analysis of UV-B light, drought and cold stress responses. Plant J. 50, 347-363. doi: 10.1111/j.1365-313X.2007.03052.X

Kotak, S., Larkindale, J., Lee, U., von Koskull-Döring, P., Vierling, E., and Scharf, K. D. (2007a). Complexity of the heat stress response in plants. Curr. Opin. Plant Biol. 10, 310-316. doi: 10.1016/j.pbi.2007.04.011

Kotak, S., Vierling, E., Bäumlein, H., and von Koskull-Döring, P. (2007b). A novel transcriptional cascade regulating expression of heat stress proteins during seed development of Arabidopsis. Plant Cell 19, 182-195. doi: 10.1105/tpc.106.048165

Langfelder, P., and Horvath, S. (2008). WGCNA: an R package for weighted correlation network analysis. BMC Bioinformatics 9:559. doi: 10.1186/14712105-9-559

Langfelder, P., Zhang, B., and Horvath, S. (2008). Defining clusters from a hierarchical cluster tree: the Dynamic Tree Cut package for R. Bioinformatics 24, 719-720. doi: 10.1093/bioinformatics/btm563

Lee, C. M., and Thomashow, M. F. (2012). Photoperiodic regulation of the Crepeat binding factor $(\mathrm{CBF})$ cold acclimation pathway and freezing tolerance in Arabidopsis thaliana. Proc. Natl. Acad. Sci. U.S.A. 109, 15054-15059. doi: 10.1073/pnas.1211295109

Li, P. S., Yu, T. F., He, G. H., Chen, M., Zhou, Y. B., Chai, S. C., et al. (2014). Genome-wide analysis of the Hsf family in soybean and functional identification of GmHsf-34 involvement in drought and heat stresses. BMC Genomics 15:1009. doi: 10.1186/1471-2164-15-1009

Li, X.-Q. (2016). Natural attributes and agricultural implications of somatic genome variation. Curr. Issues Mol. Biol. 20, 29-46.

Li, X.-Q., Xing, T., and Du, D. (2016). Identification of top-ranked proteins within a directional protein interaction network using the PageRank algorithm: applications in humans and plants. Curr. Issues Mol. Biol. 20, 13-28.

Lin, Y. X., Jiang, H. Y., Chu, Z. X., Tang, X. L., Zhu, S. W., and Cheng, B. J. (2011). Genome-wide identification, classification and analysis of heat shock transcription factor family in maize. BMC Genomics 12:76. doi: 10.1186/14712164-12-76

Lin, Z., Kong, H., Nei, M., and Ma, H. (2006). Origins and evolution of the recA/RAD51 gene family: evidence for ancient gene duplication and endosymbiotic gene transfer. Proc. Natl. Acad. Sci. U.S.A. 103, 10328-10333. doi: 10.1073/pnas.0604232103

Liu, H. C., and Charng, Y. Y. (2013). Common and distinct Functions of Arabidopsis class A1 and A2 heat shock factors in diverse abiotic stress responses and development. Plant Physiol. 163, 276-290. doi: 10.1104/pp.113.221168

Lohmann, C., Eggers-Schumacher, G., Wunderlich, M., and Schöffl, F. (2004) Two different heat shock transcription factors regulate immediate early expression of stress genes in Arabidopsis. Mol. Genet. Genomics 271, 11-21. doi: 10.1007/s00438-003-0954-8

Lyck, R., Harmening, U., Hohfeld, I., Treuter, E., Scharf, K. D., and Nover, L. (1997). Intracellular distribution and identification of the nuclear localization signals of two plant heat-stress transcription factors. Planta 202, 117-125. doi: $10.1007 / \mathrm{s} 004250050110$

Ma, J., Xu, Z. S., Wang, F., Tan, G. F., Li, M. Y., and Xiong, A. S. (2014). Genomewide analysis of HSF family transcription factors and their responses to abiotic stresses in two Chinese cabbage varieties. Acta. Physiol. Plant 36, 513-523. doi: $10.1007 / \mathrm{s} 11738-013-1432-5$

Miller, G., and Mittler, R. (2006). Could heat shock transcription factors function as hydrogen peroxide sensors in plants? Ann. Bot. 98, 279-288. doi: 10.1093/aob/mcl107

Mishra, S. K., Tripp, J., Winkelhaus, S., Tschiersch, B., Theres, K., Nover, L., et al. (2002). In the complex family of heat stress transcription factors, HsfA1 has a unique role as master regulator of thermotolerance in tomato. Genes Dev. 16, 1555-1567. doi: 10.1101/gad.228802

Mittler, R., Finka, A., and Goloubinoff, P. (2012). How do plants feel the heat? Trends Biochem. Sci. 37, 118-125. doi: 10.1016/j.tibs.2011.11.007

Morimoto, R. I. (1993). Cells in stress: transcriptional activation of heat shock genes. Science 259, 1409-1410. doi: 10.1126/science.8451637

Murashige, T., and Skoog, F. (1962). A revised medium for rapid growth and bio assays with tobacco tissue cultures. Physiol. Plant 15, 473-497. doi: 10.1111/j.1399-3054.1962.tb08052.x

Nishizawa, A., Yabuta, Y., Yoshida, E., Maruta, T., Yoshimura, K., and Shigeoka, S. (2006). Arabidopsis heat shock transcription factor A2 as a key regulator in response to several types of environmental stress. Plant J. 48, 535-547. doi: 10.1111/j.1365-313X.2006.02889.X

Nover, L., Bharti, K., Döring, P., Mishra, S. K., Ganguli, A., Scharf, K., et al. (2001). Arabidopsis and the heat stress transcription factor world: how many heat stress transcription factors do we need? Cell Stress Chaperones 6, 177-189.

PGSC (Potato Genome Sequencing Consortium) (2011). Genome sequence and analysis of the tuber crop potato. Nature 475, 189-195. doi: 10.1038 /nature 10158

Riaño-Pachón, D. M., Ruzicic, S., Dreyer, I., and Mueller-Roeber, B. (2007). PlnTFDB: an integrative plant transcription factor database. $B M C$ Bioinformatics 8:42. doi: 10.1186/1471-2105-8-42

Sakuma, Y., Maruyama, K., Qin, F., Osakabe, Y., Shinozaki, K., and YamaguchiShinozaki, K. (2006). Dual function of an Arabidopsis transcription factor DREB2A in water-stress-responsive and heat-stressresponsive gene expression. Proc. Natl. Acad. Sci. U.S.A. 103, 18822-18827. doi: 10.1073/pnas.0605639103

Scharf, K. D., Berberich, T., Ebersberger, I., and Nover, L. (2012). The plant heat stress transcription factor (Hsf) family: structure, function and evolution. Biochim. Biophys. Acta 1819, 104-119. doi: 10.1016/j.bbagrm.2011. 10.002

Schlueter, J. A., Dixon, P., Granger, C., Grant, D., Clark, L., Doyle, J. J., et al. (2004). Mining EST databases to resolve evolutionary events in major crop species. Genome 47, 868-876. doi: 10.1139/g04-047

Schöffl, F., Prändl, R., and Reindl, A. (1998). Regulation of the heat-shock response. Plant Physiol. 117, 1135-1141. doi: 10.1104/pp.117.4.1135

Shannon, P., Markiel, A., Ozier, O., Baliga, N. S., Wang, J. T., Ramage, D., et al. (2003). Cytoscape: a software environment for integrated models of biomolecular interaction networks. Genome Res. 13, 2498-2504. doi: $10.1101 /$ gr. 1239303

Singh, K., Foley, R. C., and Onate-Sanchez, L. (2002). Transcription factors in plant defense and stress responses. Curr. Opin. Plant Biol. 5, 430-436. doi: $10.1016 /$ S1369-5266(02)00289-3

Sorger, P. K., and Nelson, H. (1989). Trimerization of a yeast transcriptional activator via a coiled-coil motif. Cell 59, 807-813. doi: 10.1016/00928674(89)90604-1 
Swindell, W. R., Huebner, M., and Weber, A. P. (2007). Transcriptional profiling of Arabidopsis heat shock proteins and transcription factors reveals extensive overlap between heat and non-heat stress response pathways. BMC Genomics 8:125. doi: 10.1186/1471-2164-8-125

Thornton, J. W., and DeSalle, R. (2000). Gene family evolution and homology: genomics meets phylogenetics. Annu. Rev. Genomics Hum. Genet. 1, 41-73. doi: 10.1146/annurev.genom.1.1.41

Tsvetkova, N. M., Horvath, I., Torok, Z., Wolkers, W. F., Balogi, Z., Shigapova, N., et al. (2002). Small heat-shock proteins regulate membrane lipid polymorphism. Proc. Natl. Acad. Sci. U.S.A. 99, 13504-13509. doi: 10.1073/pnas.192468399

Von Koskull-Döring, P., Scharf, K. D., and Nover, L. (2007). The diversity of plant heat stress transcription factors. Trends Plant Sci. 12, 452-457. doi: 10.1016/j.tplants.2007.08.014

Wang, C., Zhang, Q., and Shou, H. X. (2009). Identification and expression analysis of OsHsfs in rice. J. Zhejiang Univ. Sci. B 10, 291-300. doi: 10.1631/jzus.B0820190

Wang, F., Dong, Q., Jiang, H., Zhu, S., Chen, B., and Xiang, Y. (2012). Genomewide analysis of the heat shock transcription factors in Populus trichocarpa and Medicago truncatula. Mol. Biol. Rep. 39, 1877-1886. doi: 10.1007/s11033-0110933-9

Wang, M., Vannozzi, A., Wang, G., Liang, Y. H., Tornielli, G. B., Zenoni, S., et al. (2014). Genome and transcriptome analysis of the grapevine (Vitis vinifera L.) WRKY gene family. Hortic. Res. 1, 1-16. doi: 10.1038/hortres.2014.16

Wiederrecht, G., Seto, D., and Parker, C. S. (1988). Isolation of the gene encoding the S. cerevisiae heat shock transcription factor. Cell 54, 841-853. doi: 10.1016/S0092-8674(88)91197-X

$\mathrm{Wu}$, C. (1995). Heat shock transcription factors: structure and regulation. Annu. Rev. Cell Dev. Biol. 11, 441-469. doi: 10.1146/annurev.cb.11.110195.002301

Xue, G. P., Sadat, S., Drenth, J., and McIntyre, C. L. (2014). The heat shock factor family from Triticum aestivum in response to heat and other major abiotic stresses and their role in regulation of heat shock protein genes. J. Exp. Bot. 65, 539-557. doi: 10.1093/jxb/ert399

Yang, L., Xie, C., Li, W., Zhang, R., Jue, D., and Yang, Q. (2013). Expression of a wild eggplant ribosomal protein L13a in potato enhances resistance to Verticillium dahlia. Plant Cell Tiss. Organ Cult. 115, 329-340. doi: 10.1007/s11240-0130365-4

Yoshida, T., Ohama, N., Nakajima, J., Kidokoro, S., Mizoi, J., Nakashima, K., et al. (2011). Arabidopsis HsfA1 transcription factors function as the main positive regulators in heat shock-responsive gene expression. Mol. Genet. Genomics 286, 321-332. doi: 10.1007/s00438-011-0647-7
Yoshida, T., Sakuma, Y., Todaka, D., Maruyama, K., Qin, F., Mizoi, J., et al. (2008). Functional analysis of an Arabidopsis heat-shock transcription factor $\mathrm{HsfA} 3$ in the transcriptional cascade downstream of the DREB2A stressregulatory system. Biochem. Biophys. Res. Commun. 368, 515-521. doi: 10.1016/j.bbrc.2008.01.134

Yuan, S. X., Xu, B., Zhang, J., Xie, Z. N., Cheng, Q., Yang, Z. M., et al. (2015). Comprehensive analysis of CCCH-type zinc finger family genes facilitates functional gene discovery and reflects recent allopolyploidization event in tetraploid switchgrass. BMC Genomics 16:129. doi: 10.1186/s12864-0151328-4

Zhang, J., Liu, B., Li, J. B., Zhang, L., Wang, Y., Zheng, H. Q., et al. (2015). Hsf and Hsp gene families in Populus: genomewide identification, organization and correlated expression during development and in stress responses. BMC Genomics 16:181. doi: 10.1186/s12864-0151398-3

Zheng, J. Y., Li, D. J., Cheng, X. Y., Yang, Y. H., and Xie, B. X. (2008). The evolution types and function of animal heat shock transcription factors. Biotechnol. Bull. 17-24.

Zhou, S. J., Zhang, P., Jing, Z., and Shi, J. L. (2013). Genome-wide identification and analysis of heat shock transcription factor family in cucumber (Cucumis sativus L.). Plant Omics 6, 449-455.

Zhu, X., Thalor, S. K., Takahashi, Y., Berberich, T., and Kusano, T. (2012). An inhibitory effect of the sequence-conserved upstream open-reading frame on the translation of the main open-reading frame of HsfB1 transcripts in Arabidopsis. Plant Cell Environ. 35, 2014-2030. doi: 10.1111/j.13653040.2012.02533.x

Conflict of Interest Statement: The authors declare that the research was conducted in the absence of any commercial or financial relationships that could be construed as a potential conflict of interest.

The reviewer SG and handling Editor declared their shared affiliation, and the handling Editor states that the process nevertheless met the standards of a fair and objective review.

Copyright (c) 2016 Tang, Zhu, Song, Lin, Cai, Wang and Yang. This is an open-access article distributed under the terms of the Creative Commons Attribution License (CC $B Y)$. The use, distribution or reproduction in other forums is permitted, provided the original author(s) or licensor are credited and that the original publication in this journal is cited, in accordance with accepted academic practice. No use, distribution or reproduction is permitted which does not comply with these terms. 